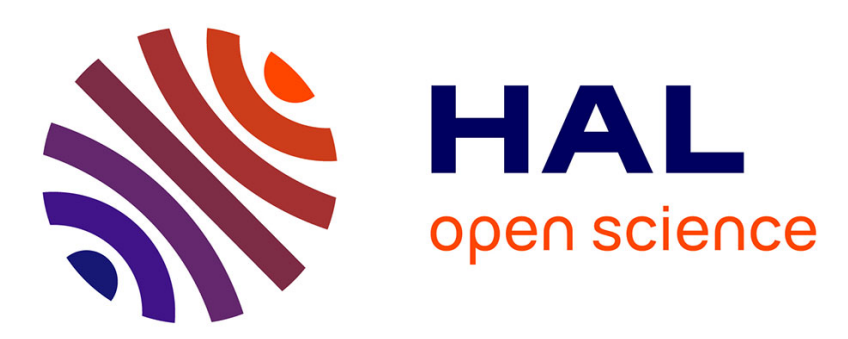

\title{
Multislope MUSCL method for general unstructured meshes
}

\author{
Clément Le Touze, Angelo Murrone, Herve Guillard
}

\section{To cite this version:}

Clément Le Touze, Angelo Murrone, Herve Guillard. Multislope MUSCL method for general unstructured meshes. Journal of Computational Physics, 2014, pp.44. 10.1016/j.jcp.2014.12.032 . hal$00939475 \mathrm{v} 3$

\section{HAL Id: hal-00939475 \\ https://hal.inria.fr/hal-00939475v3}

Submitted on 9 Jan 2015

HAL is a multi-disciplinary open access archive for the deposit and dissemination of scientific research documents, whether they are published or not. The documents may come from teaching and research institutions in France or abroad, or from public or private research centers.
L'archive ouverte pluridisciplinaire HAL, est destinée au dépôt et à la diffusion de documents scientifiques de niveau recherche, publiés ou non, émanant des établissements d'enseignement et de recherche français ou étrangers, des laboratoires publics ou privés. 


\title{
Multislope MUSCL method for general unstructured meshes
}

\author{
C. Le Touze ${ }^{\mathrm{a}, *}$, A. Murrone ${ }^{\mathrm{a}}$, H. Guillard ${ }^{\mathrm{b}, \mathrm{c}}$ \\ ${ }^{a}$ ONERA - The French Aerospace Lab, F-92322 Châtillon, France \\ ${ }^{b}$ INRIA, BP 9306902 Sophia Antipolis Cedex, France \\ ${ }^{c}$ University of Nice, Laboratoire J.A. Dieudonne, Parc Valrose, 06108 Nice Cedex 2, France
}

\begin{abstract}
The multislope concept has been recently introduced in the literature to deal with MUSCL reconstructions on triangular and tetrahedral unstructured meshes in the finite volume cell-centered context. Dedicated scalar slopes are used to compute the interpolations on each face of a given element, in opposition to the monoslope methods in which a unique limited gradient is used. The multislope approach reveals less expensive and potentially more accurate than the classical gradient techniques. Besides, it may also help the robustness when dealing with hyperbolic systems involving complex solutions, with large discontinuities and high density ratios. However some important limitations on the mesh topology still have to be overcome with the initial multislope formalism. In this paper, a generalized multislope MUSCL method is introduced for cell-centered finite volume discretizations. The method is freed from constraints on the mesh topology, thereby operating on completely general unstructured meshes. Moreover optimal second-order accuracy is reached at the faces centroids. The scheme can be written with nonnegative coefficients, which makes it $\mathrm{L}^{\infty}$-stable. Special attention has also been paid to equip the reconstruction procedure with well-adapted dedicated limiters, potentially CFL-dependent. Numerical tests are provided to prove the ability of the method to deal with completely general meshes, while exhibiting second-order accuracy.
\end{abstract}

Keywords: cell-centered finite volume method, general unstructured meshes, multislope MUSCL technique, CFL-dependent limiters

\section{Introduction}

Since its introduction by Van Leer [1], the MUSCL method has become a standard widely used in industrial production finite volume codes [2, 3]. As a sequel to Godunov's method for the approximation of the convective fluxes [4], it increases the scheme order while preserving its stability $[1,5]$. In spite of its lower accuracy compared with ENO, WENO, or Discontinuous Galerkin methods, the MUSCL technique is much more afford15 able and easier to implement, especially when dealing with complex systems of equations on $3 \mathrm{D}$ general unstructured meshes.

\footnotetext{
* Corresponding author

Email address: clement.le_touze@onera.fr (C. Le Touze )

Preprint submitted to Elsevier
}

January 8, 2015 
Initially, Van Leer's method was dedicated to mono-dimensional scalar conservation equations, on regular grids. The principle is as follows: new approximations of the variables are interpolated from the cell centers towards the cell edges, while the expression

20 of the numerical flux at the interface is unchanged. Upward and backward slopes are used to compute the interpolations in a way that prevents spurious oscillations [6] and preserves the stability of the scheme through the Total Variation Diminishing (TVD) property [7].

Extension to multidimensional configurations has first consisted in applying the 25 MUSCL procedure to each direction for structured grids [8]. However, the work of Goodman and LeVeque [9] proved that only a first-order scheme could be obtained under the TVD restriction. This was overcome with the positive coefficients schemes developed by Spekreijse [10], and the introduction of the Local Maximum Principle. Generalization to unstructured meshes was made possible thanks to the Local Extremum Diminishing

30 (LED) concept [11]. Eventually, a whole range of MUSCL methods is available to deal with multidimensional unstructured grids. These depend on how the slopes are both computed and limited.

On the one hand, there exists a large family of monoslope methods: a unique gradient is used to reconstruct a linear interpolation of the solution within a given cell $[12,3]$.

35 This linear reconstruction (possibly limited) is then used to compute the fluxes at the cell interfaces. In contrast to these methods, a "face by face" approach has been introduced in the cell-vertex context in [13] (see also [14]): upstream and downstream triangles were sought to compute the slopes. In these multislope methods, every reconstruction on a cell face is computed with its own limited scalar slope. This last kind of approach has

40 been recently examined in the cell-centered formulation [15], and adapted to the cellvertex formalism in [16]. Classical limiters slightly modified to take into account the local heterogeneity of the mesh are used to limit the different slopes [17]. The multislope approach appears to be robust and able to attain better accuracy than its monoslope counterpart. See for instance [18] for an application of this method to two-phase flow 45 simulation with high density ratios.

However, the technique introduced in [15] for the cell-centered formalism exhibits some limitations. First, restrictive hypotheses on the mesh topology are made. The technique of [15] indeed only applies to triangular or tetrahedral meshes, and consequently cannot deal with general polyhedral grids. Moreover, two versions of the method

50 are considered depending on the point where the interpolation is computed on the face. The most accurate version is when the face centroid is used since it leads to the best fluxes approximation. Unfortunately, the stability of the scheme cannot be guaranteed with this version [15]. Inspired by the above-mentioned previous works, we introduce in this paper a new multislope technique to overcome these two major limitations.

${ }_{55}$ First, the method we design is free from any restriction on the mesh topology or regularity. This is important for some industrial applications since the meshes used by several production codes are completely general unstructured meshes where the cells are made of arbitrary polyhedral objects. This is for instance the case of the CEDRE code developed at ONERA, which is a multi-physics software for energetics [19] based on the

60 cell-centered finite volume method. The use of arbitrary polygonal meshes is also common in the gas and petroleum industry for reservoir simulations and for instance the ARCANE [20] development platform of CEA-IFPEN is designed to handle 3D meshes composed of several types of polyhedral entities (tetrahedra, prisms, hexahedra, octahedra, ...). 
Second, we come up with a method reconstructing the values at the faces centroids and complying with the maximum principle. This ensures that the method is able to reach second order accuracy on smooth solutions with no sacrifice of robustness issues.

This paper is organized as follows. In section 2, we introduce the MUSCL strategy for finite volume discretization of scalar hyperbolic conservation laws in the cell-centered formulation, and for general unstructured meshes. The following sections describe the 70 successive steps of our new MUSCL reconstruction procedure. We explain in section 3 how to compute the slopes for general unstructured grids, in two-dimensional or threedimensional space. Then, essential properties of the scheme are proved in section 4 , namely second-order consistency and $\mathrm{L}^{\infty}$-stability. Section 5 is devoted to the design of accurate potentially CFL-dependent limiters. Finally, numerical results are provided in

75 section 6 to assess the performance of the method on general grids.

\section{Framework of the MUSCL methods}

\subsection{Finite volume discretization of scalar hyperbolic conservation laws}

To introduce the MUSCL methods for finite volume schemes, we herein consider a scalar hyperbolic conservation equation (1) associated to the initial and boundary 80 conditions (2). Let $\Omega \subset \mathbb{R}^{3}$ be a bounded domain with the boundary $\partial \Omega$. Let us consider $u(\boldsymbol{x}, t)$ and $\boldsymbol{F}[\boldsymbol{x}, t, u(\boldsymbol{x}, t)]=\boldsymbol{v}(\boldsymbol{x}, t) u(\boldsymbol{x}, t)$, respectively $\mathbb{R}$ and $\mathbb{R}^{3}$-valued functions, with $\boldsymbol{x} \in \Omega$ and $t \in[0, T]$. The function $u_{0}(\boldsymbol{x})$ is the initial solution, whereas $u_{b}\left(\boldsymbol{x} \in \partial \Omega^{-}(t), t\right)$ is the Dirichlet boundary condition. Note that the flux function $\boldsymbol{F}$ depends a priori on position $\boldsymbol{x}$ and time $t$ in addition to the scalar function $u$. But from here on, this

85 dependency will not be explicitly written. For the sake of simplicity, the flux will be therefore either written $\boldsymbol{F}(u)$ or even only $\boldsymbol{F}$ depending on the context.

$$
\begin{gathered}
\left.\partial_{t} u(\boldsymbol{x}, t)+\nabla \cdot \boldsymbol{F}[\boldsymbol{x}, t, u(\boldsymbol{x}, t)]=0 \quad \boldsymbol{x} \in \Omega, \quad t \in\right] 0, T[ \\
u(\boldsymbol{x}, t=0)=u_{0}(\boldsymbol{x}), \quad \boldsymbol{x} \in \Omega \quad \text { and } \quad u\left(\boldsymbol{x} \in \partial \Omega^{-}(t), t\right)=u_{b}\left(\boldsymbol{x} \in \partial \Omega^{-}(t), t\right)
\end{gathered}
$$

In $(2), \partial \Omega^{-}(t)=\{\boldsymbol{x} \in \partial \Omega \mid \boldsymbol{v}(\boldsymbol{x}, t) \cdot \boldsymbol{n}(\boldsymbol{x})<0\}, \boldsymbol{v}(\boldsymbol{x}, t)$ and $\boldsymbol{n}(\boldsymbol{x})$ are the velocity field and the outwards normal vector on the boundary respectively. Let us assume the domain $\Omega$ 90 polygonal, then we introduce a discretization of $\Omega$ made up with general polyhedra $K_{i}$ defined by an arbitrary number of faces (see notations on Figure 1 for a $2 \mathrm{D}$ configuration). We denote by $\mathcal{V}(i)$ the vicinity of the element $K_{i}$, defined as the set of neighboring elements $K_{j}$ with a common face $S_{i j}=K_{i} \cap K_{j}$. By extension, $\mathcal{V}(i)$ will sometimes also refer to the set of indexes $j$ of the neighboring elements $K_{j}$.

${ }_{95} \quad$ Any face $S_{i j}$ is defined by an arbitrary number $m_{i j}$ of vertices $\boldsymbol{P}_{i j, k}$, with $k \in\left[1, m_{i j}\right]$. As a result, faces are not necessarily planar in $3 \mathrm{D}$. But we assume that each face $S_{i j}$ is associated with a geometrical point $\boldsymbol{M}_{i j}$ such that $S_{i j}$ is a triangulation lying on the polygonal contour $\boldsymbol{P}_{i j, k}$, with $k \in\left[1, m_{i j}\right]$, and with $\boldsymbol{M}_{i j}$ as common point. $\boldsymbol{M}_{i j}$ is defined as the gravity center of the triangulated face according to

$$
\sum_{k=1}^{m_{i j}}\left|S_{i j, k}\right| \overrightarrow{\boldsymbol{M}_{i j} \boldsymbol{P}_{i j, k}}=\mathbf{0},
$$


100 where $\left|S_{i j, k}\right|$ is the area of the triangle $\boldsymbol{M}_{i j} \boldsymbol{P}_{i j, k} \boldsymbol{P}_{i j, k+1}$ with cycle order. Note that (3) is a non-linear equation for $\boldsymbol{M}_{i j}$ which is solved in practice by a small number of fixed point iterations. In the sequel, we will denote by $\left|S_{i j}\right|$ the surface area of the face $S_{i j}$, which is defined as the sum of the areas of the triangles $\boldsymbol{M}_{i j} \boldsymbol{P}_{i j, k} \boldsymbol{P}_{i j, k+1}$. The unit normal vector $\boldsymbol{n}_{i j}$ of the face $S_{i j}$ (from $K_{i}$ to $K_{j}$ ) is defined as the sum of the unit 105 normal vectors of each triangle weighted by its surface area. With this definition of the cell faces, it is possible to define the volume $\left|K_{i}\right|$ and the centroid $\boldsymbol{B}_{i}$ of the cell $K_{i}$ by

$$
\left|K_{i}\right|=\int_{K_{i}} d V, \quad \boldsymbol{B}_{i}=\frac{1}{\left|K_{i}\right|} \int_{K_{i}} \boldsymbol{X} d V .
$$

Subsequently we have with second-order accuracy:

$$
\int_{K_{i}} u(\boldsymbol{x}) d V \approx\left|K_{i}\right| u\left(\boldsymbol{B}_{i}\right), \quad \int_{S_{i j}} u(\boldsymbol{x}) d S \approx\left|S_{i j}\right| u\left(\boldsymbol{M}_{i j}\right) .
$$

For more practical details on the computation of the geometrical quantities associated to a cell, see [21] or [22].

Let $\left(t^{n}\right)_{n \in[0, N]}$ be a discretization of time, and $\Delta t=t^{n+1}-t^{n}$ be the time step. We use a cell-centered formulation, so at the time $t^{n}, U_{i}^{n}$ stands for the value of $u$ at the cell centroid $\boldsymbol{B}_{i}$, with a second-order accuracy. Using the explicit Euler method in time, applying the divergence theorem, and after integration over the control volumes $K_{i}$, the first-order finite volume scheme reads in

$$
\left|K_{i}\right| \frac{U_{i}^{n+1}-U_{i}^{n}}{\Delta t}+\sum_{j \in \mathcal{V}(i)} \int_{S_{i j}} \boldsymbol{F} \cdot \boldsymbol{n}_{i j} d S=0 .
$$

115 We then introduce the numerical flux function $\phi_{i j}\left(U_{i}^{n}, U_{j}^{n}, \boldsymbol{n}_{i j}\right)$ whose purpose is to approximate the flux integral over the face $S_{i j}$ as follows:

$$
\phi_{i j}\left(U_{i}^{n}, U_{j}^{n}, \boldsymbol{n}_{i j}\right)\left|S_{i j}\right| \simeq \int_{S_{i j}} \boldsymbol{F} \cdot \boldsymbol{n}_{i j} d S
$$

and rewrite the scheme

$$
U_{i}^{n+1}=U_{i}^{n}-\Delta t \sum_{j \in \mathcal{V}(i)} \frac{\left|S_{i j}\right|}{\left|K_{i}\right|} \phi_{i j}\left(U_{i}^{n}, U_{j}^{n}, \boldsymbol{n}_{i j}\right) .
$$

The numerical flux has to respect some properties to ensure the stability of the scheme under an appropriate CFL condition [2]. Indeed, $\phi_{i j}$ must be a monotonous $\mathcal{C}^{1}$ function, consistent with the physical flux (see section 4.2.1). Various numerical flux functions can be chosen. For applications in energetics, where high density ratios and discontinuities are encountered, upwind numerical fluxes are mostly used for the sake of robustness. However, centered fluxes are sometimes also used, but they require that some artificial diffusion is introduced so that the scheme remains stable.

Nevertheless, it is well-known that upwind schemes bring about numerical diffusion, which can be reduced using the MUSCL strategy. New arguments are provided to the numerical flux function, without modification of the finite volume scheme. The cellcentered states $U_{i}^{n}$ and $U_{j}^{n}$ are replaced respectively by interpolated states $U_{i j}^{n}$ and $U_{j i}^{n}$ 
on the faces $S_{i j}$. We now drop the dependency of the numerical flux on the normal vector $\boldsymbol{n}_{i j}$ for the sake of simplicity. The MUSCL scheme then reads in

$$
U_{i}^{n+1}=U_{i}^{n}-\Delta t \sum_{j \in \mathcal{V}(i)} \frac{\left|S_{i j}\right|}{\left|K_{i}\right|} \phi_{i j}\left(U_{i j}^{n}, U_{j i}^{n}\right) .
$$

The whole difficulty, especially with unstructured grids, lies in how to compute the interpolations so as to get second-order accuracy while still complying with the Discrete Maximum Principle (DMP) defined below, which ensures that the scheme is $\mathrm{L}^{\infty}$-stable.

Definition 1. The MUSCL scheme written in (9) complies with the DMP if for any element $K_{i}$, the updated value at time $t^{n+1}$ is bounded by the minimum and maximum values at time $t^{n}$ within $K_{i}$ and a given neighborhood $\mathcal{U}\left(K_{i}\right)$, which can be a priori of arbitrary extent. Let $\overline{\mathcal{U}_{i}}$ denote the union $\mathcal{U}\left(K_{i}\right) \cup\left\{K_{i}\right\}$, then the DMP formally reads in

$$
\forall K_{i} \in \Omega, \quad \min _{K_{j} \in \overline{\mathcal{U}}_{i}} U_{j}^{n} \leq U_{i}^{n+1} \leq \max _{K_{j} \in \overline{\mathcal{U}}_{i}} U_{j}^{n}
$$

\subsection{New multislope method for general unstructured meshes}

A new multislope reconstruction procedure is introduced in this section, operating on general unstructured meshes and computing the reconstruted values at the face centroids $\boldsymbol{M}_{i j}$. The technique generalizes the multislope method introduced in [15, 23, 17], therefore similar notations are adopted. As in the above-cited technique, and as in the original MUSCL method, both a backward and a forward scalar slopes, respectively written $p_{i j}^{-}$ and $p_{i j}^{+}$, are computed for each face $S_{i j}$ of a given element $K_{i}$. In a classical way, we introduce $r_{i j}=p_{i j}^{-} / p_{i j}^{+}$, and use a limiter function $\varphi\left(r_{i j}\right)$, which turns these slopes into one limited slope to ensure that no unphysical oscillation is introduced. Therefore, the reconstructed values read as follows:

$$
\begin{aligned}
& U_{i j}=U_{i}+p_{i j}^{+} \varphi\left(r_{i j}, \mathcal{G}_{i j}\right)\left\|\boldsymbol{B}_{i} \boldsymbol{M}_{i j}\right\|, \\
& U_{j i}=U_{j}+p_{j i}^{+} \varphi\left(r_{j i}, \mathcal{G}_{j i}\right)\left\|\boldsymbol{B}_{j} \boldsymbol{M}_{i j}\right\|,
\end{aligned}
$$

where $\mathcal{G}_{i j}$ is a set of geometrical parameters, which will be defined.

In the following sections, we first give the procedure used to compute the forward and backward slopes for general unstructured meshes. Afterwards we prove some essential properties of the new reconstruction procedure, such as second-order consistency and $\mathrm{L}^{\infty}$-stability. We finally address the issue of the limiters functions to be used, with a special effort to derive a well-adapted limiter for our new reconstruction technique.

\section{Building the slopes}

In addition to the "face-to-face" neighborhood $\mathcal{V}(i)$, we now define the $\mathcal{W}(i)$ neighborhood as the set of elements sharing at least a vertex with $K_{i}$. Note that $\mathcal{V}(i) \subset \mathcal{W}(i)$. Let us consider the system formed by the element $K_{i}$ of centroid $\boldsymbol{B}_{i}$, and the face $S_{i j}$ of centroid $\boldsymbol{M}_{i j}$, for which we want to compute both a forward and a backward slope. The principle is to determine two points $\boldsymbol{H}_{i j}^{-}$and $\boldsymbol{H}_{i j}^{+}$, both located on the axis $\left(\boldsymbol{B}_{i} \boldsymbol{M}_{i j}\right)$, 
respectively backward and forward the point $\boldsymbol{B}_{i}$ (see Figure 1). These points are a priori neither vertices of the grid, nor elements centroids. However, the way they are defined makes it possible to evaluate second-order consistent values of the variables, using a wellchosen set of neighboring elements. Eventually, the computed values $U_{H_{i j}^{-}}$and $U_{H_{i j}^{+}}$at points $\boldsymbol{H}_{i j}^{-}$and $\boldsymbol{H}_{i j}^{+}$, are used to compute the backward and forward slopes as follows:

$$
p_{i j}^{+}=\frac{U_{H_{i j}^{+}}-U_{i}}{\left\|\boldsymbol{B}_{i} \boldsymbol{H}_{i j}^{+}\right\|}, \quad p_{i j}^{-}=\frac{U_{i}-U_{H_{i j}^{-}}}{\left\|\boldsymbol{B}_{i} \boldsymbol{H}_{i j}^{-}\right\|} .
$$

The process to determine the points $\boldsymbol{H}_{i j}^{-}$and $\boldsymbol{H}_{i j}^{+}$, and then to get the values $U_{H_{i j}^{-}}$ and $U_{H_{i j}^{+}}$, depends on the dimension of the domain $\Omega$.

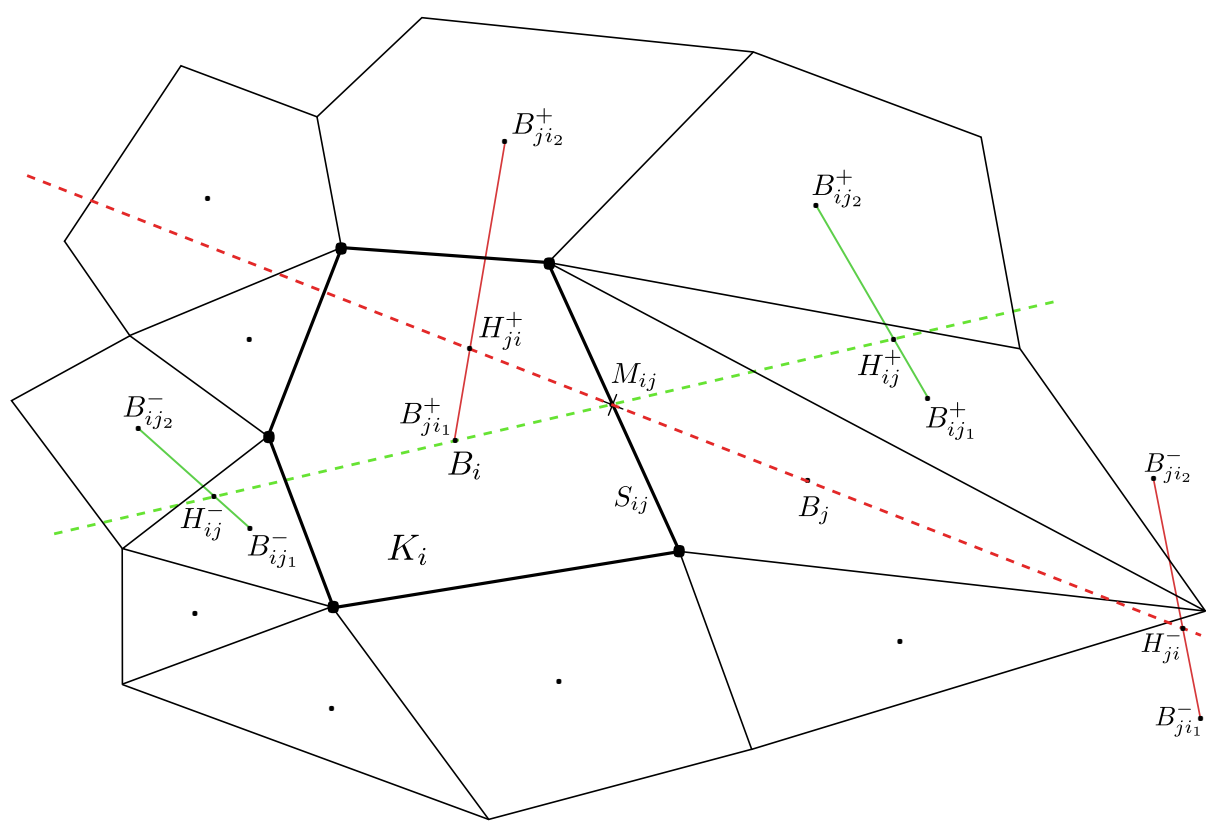

Figure 1: Forward and backward points $\boldsymbol{H}_{i j}^{+}$and $\boldsymbol{H}_{i j}^{-}$in the $2 \mathrm{D}$ configuration.

\subsection{Procedure for 2D configurations}

Let $K_{i j_{1}}^{-}$in $\mathcal{W}(i)$ be the most backward neighboring element of $K_{i}$ with respect to the direction $\left(\boldsymbol{B}_{i} \boldsymbol{M}_{i j}\right)$, in the sense that

$$
\cos \left(\boldsymbol{B}_{i j_{1}}^{-} \boldsymbol{B}_{i}, \boldsymbol{B}_{i} \boldsymbol{M}_{i j}\right)=\max _{k \in \mathcal{W}(i)} \cos \left(\boldsymbol{B}_{k} \boldsymbol{B}_{i}, \boldsymbol{B}_{i} \boldsymbol{M}_{i j}\right)
$$

Let $K_{i j_{2}}^{-}$in $\mathcal{W}(i)$ be the following most backward element, provided that it is located on the other side of the axis $\left(\boldsymbol{B}_{i} \boldsymbol{M}_{i j}\right)$. In other words, $K_{i j_{2}}^{-}$is such that

$$
\cos \left(\boldsymbol{B}_{i j_{2}}^{-} \boldsymbol{B}_{i}, \boldsymbol{B}_{i} \boldsymbol{M}_{i j}\right)=\max _{\substack{k \in \overline{\mathcal{W}}(i) \\ 6}} \cos \left(\boldsymbol{B}_{k} \boldsymbol{B}_{i}, \boldsymbol{B}_{i} \boldsymbol{M}_{i j}\right)
$$


with

$$
\overline{\mathcal{W}}(i)=\left\{k \mid k \in \mathcal{W}(i), K_{k} \neq K_{i j_{1}}^{-}, \sin \left(\boldsymbol{B}_{k} \boldsymbol{B}_{i}, \boldsymbol{B}_{i} \boldsymbol{M}_{i j}\right) \sin \left(\boldsymbol{B}_{i j_{1}}^{-} \boldsymbol{B}_{i}, \boldsymbol{B}_{i} \boldsymbol{M}_{i j}\right) \leq 0\right\} .
$$

Then, we can define the intersection point $\boldsymbol{H}_{i j}^{-}$between the axis $\left(\boldsymbol{B}_{i} \boldsymbol{M}_{i j}\right)$ and the line $\left(\boldsymbol{B}_{i j_{1}}^{-} \boldsymbol{B}_{i j_{2}}^{-}\right)$, such that $\boldsymbol{H}_{i j}^{-}$is located inside the segment $\left[\boldsymbol{B}_{i j_{1}}^{-} \boldsymbol{B}_{i j_{2}}^{-}\right]$. Consequently, $\boldsymbol{H}_{i j}^{-}$has nonnegative barycentric coordinates $\left(\beta_{i j_{1}}^{-}, \beta_{i j_{2}}^{-}\right)$with respect to $\left(\boldsymbol{B}_{i j_{1}}^{-}, \boldsymbol{B}_{i j_{2}}^{-}\right)$, and

$$
\beta_{i j_{1}}^{-}=\frac{\left\|\boldsymbol{B}_{i j_{2}}^{-} \boldsymbol{H}_{i j}^{-}\right\|}{\left\|\boldsymbol{B}_{i j_{1}}^{-} \boldsymbol{B}_{i j_{2}}^{-}\right\|} \geq 0, \quad \beta_{i j_{2}}^{-}=\frac{\left\|\boldsymbol{B}_{i j_{1}}^{-} \boldsymbol{H}_{i j}^{-}\right\|}{\left\|\boldsymbol{B}_{i j_{1}}^{-} \boldsymbol{B}_{i j_{2}}^{-}\right\|} \geq 0, \quad \beta_{i j_{1}}^{-}+\beta_{i j_{2}}^{-}=1 .
$$

In a symmetric way, one can determine for the forward direction: $K_{i j_{1}}^{+}, K_{i j_{2}}^{+}$, the intersection point $\boldsymbol{H}_{i j}^{+}$, and its nonnegative barycentric coordinates $\left(\beta_{i j_{1}}^{+}, \beta_{i j_{2}}^{+}\right)$, such that

$$
\beta_{i j_{1}}^{+}=\frac{\left\|\boldsymbol{B}_{i j_{2}}^{+} \boldsymbol{H}_{i j}^{+}\right\|}{\left\|\boldsymbol{B}_{i j_{1}}^{+} \boldsymbol{B}_{i j_{2}}^{+}\right\|} \geq 0, \quad \beta_{i j_{2}}^{+}=\frac{\left\|\boldsymbol{B}_{i j_{1}}^{+} \boldsymbol{H}_{i j}^{+}\right\|}{\left\|\boldsymbol{B}_{i j_{1}}^{+} \boldsymbol{B}_{i j_{2}}^{+}\right\|} \geq 0, \quad \beta_{i j_{1}}^{+}+\beta_{i j_{2}}^{+}=1 .
$$

These coordinates are subsequently used to compute the variable values at points $\boldsymbol{H}_{i j}^{-}$and $\boldsymbol{H}_{i j}^{+}$according to simple weighted means:

$$
U_{H_{i j}^{-}}=\beta_{i j_{1}}^{-} U_{i j_{1}}^{-}+\beta_{i j_{2}}^{-} U_{i j_{2}}^{-}, \quad U_{H_{i j}^{+}}=\beta_{i j_{1}}^{+} U_{i j_{1}}^{+}+\beta_{i j_{2}}^{+} U_{i j_{2}}^{+},
$$

with $U_{i j_{1}}^{-}, U_{i j_{2}}^{-}, U_{i j_{1}}^{+}$, and $U_{i j_{2}}^{+}$, being the values at the cell centers $\boldsymbol{B}_{i j_{1}}^{-}, \boldsymbol{B}_{i j_{2}}^{-}, \boldsymbol{B}_{i j_{1}}^{+}$, and $\boldsymbol{B}_{i j_{2}}^{+}$. For non linear functions, the shorter the distances $\left\|\boldsymbol{B}_{i j_{1}}^{-} \boldsymbol{B}_{i j_{2}}^{-}\right\|$and $\left\|\boldsymbol{B}_{i j_{1}}^{+} \boldsymbol{B}_{i j_{2}}^{+}\right\|$, the lower the approximation error in the values $U_{H_{i j}^{-}}$and $U_{H_{i j}^{+}}$. This is why the most backward and forward neighbors are selected.

\subsection{Procedure for $3 D$ configurations}

Extension to 3D configurations is not straighforward. The difference is that we need sets of three neighboring elements instead of two, to determine the forward and backward points. For instance, when considering the backward direction, one needs to find $K_{i j_{1}}^{-}$, $K_{i j_{2}}^{-}$and $K_{i j_{3}}^{-}$, such that they define a plane that intersects the line $\left(\boldsymbol{B}_{i} \boldsymbol{M}_{i j}\right)$ inside the triangle of vertices $\boldsymbol{B}_{i j_{1}}^{-}, \boldsymbol{B}_{i j_{2}}^{-}$and $\boldsymbol{B}_{i j_{3}}^{-}$(see Figure 2 for the forward side). The intersection point $\boldsymbol{H}_{i j}^{-}$has therefore unique nonnegative normalized barycentric coordinates with respect to $\left(\boldsymbol{B}_{i j_{1}}^{-}, \boldsymbol{B}_{i j_{2}}^{-}, \boldsymbol{B}_{i j_{3}}^{-}\right)$. We denote these coordinates $\beta_{i j_{1}}^{-}, \beta_{i j_{2}}^{-}, \beta_{i j_{3}}^{-}$, which are given as ratios of surface areas:

$$
\begin{gathered}
\beta_{i j_{1}}^{-}=\frac{\mathcal{A}\left(\boldsymbol{H}_{i j}^{-} \boldsymbol{B}_{i j_{2}}^{-} \boldsymbol{B}_{i j_{3}}^{-}\right)}{\mathcal{A}\left(\boldsymbol{B}_{i j_{1}}^{-} \boldsymbol{B}_{i j_{2}}^{-} \boldsymbol{B}_{i j_{3}}^{-}\right)}, \quad \beta_{i j_{2}}^{-}=\frac{\mathcal{A}\left(\boldsymbol{H}_{i j}^{-} \boldsymbol{B}_{i j_{1}}^{-} \boldsymbol{B}_{i j_{3}}^{-}\right)}{\mathcal{A}\left(\boldsymbol{B}_{i j_{1}}^{-} \boldsymbol{B}_{i j_{2}}^{-} \boldsymbol{B}_{i j_{3}}^{-}\right)}, \quad \beta_{i j_{3}}^{-}=\frac{\mathcal{A}\left(\boldsymbol{H}_{i j}^{-} \boldsymbol{B}_{i j_{1}}^{-} \boldsymbol{B}_{i j_{2}}^{-}\right)}{\mathcal{A}\left(\boldsymbol{B}_{i j_{1}}^{-} \boldsymbol{B}_{i j_{2}}^{-} \boldsymbol{B}_{i j_{3}}^{-}\right)}, \\
\beta_{i j_{1}}^{-} \geq 0, \quad \beta_{i j_{2}}^{-} \geq 0, \quad \beta_{i j_{3}}^{-} \geq 0, \quad \beta_{i j_{1}}^{-}+\beta_{i j_{2}}^{-}+\beta_{i j_{3}}^{-}=1 .
\end{gathered}
$$

The value at the point $\boldsymbol{H}_{i j}^{-}$is obtained as follows:

$$
U_{H_{i j}^{-}}=\beta_{i j_{1}}^{-} U_{i j_{1}}^{-}+\beta_{i j_{2}}^{-} U_{i j_{2}}^{-}+\beta_{i j_{3}}^{-} U_{i j_{3}}^{-} .
$$


The symmetric procedure applied to the forward direction leads to

$$
U_{H_{i j}^{+}}=\beta_{i j_{1}}^{+} U_{i j_{1}}^{+}+\beta_{i j_{2}}^{+} U_{i j_{2}}^{+}+\beta_{i j_{3}}^{+} U_{i j_{3}}^{+} .
$$

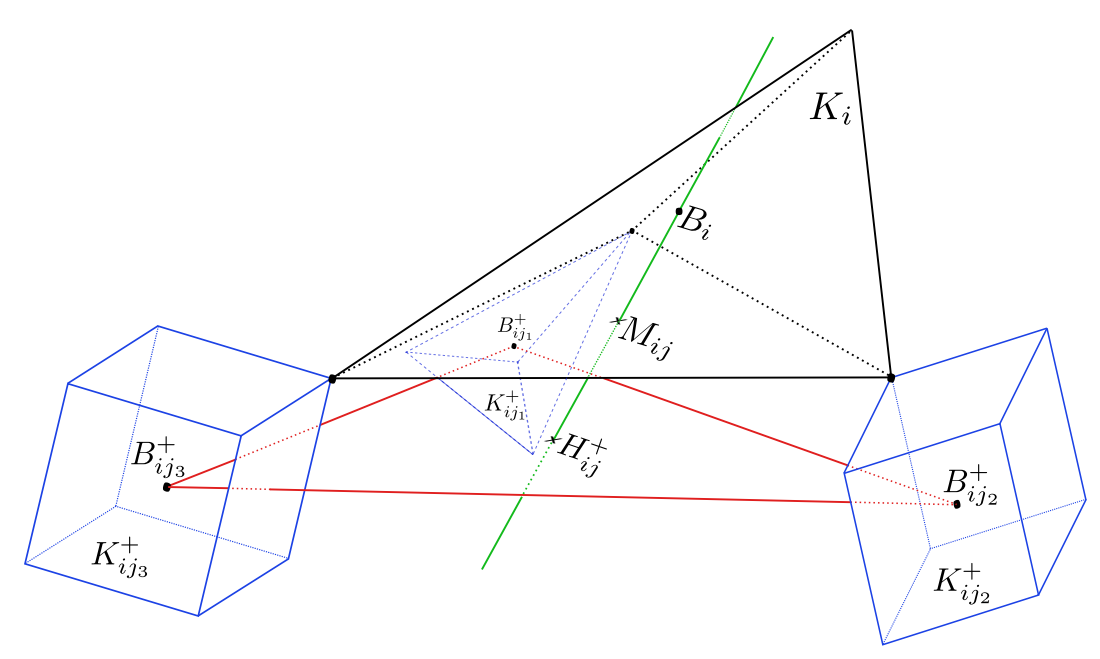

Figure 2: Forward point $\boldsymbol{H}_{i j}^{+}$for the 3D configuration.

The algorithm used to determine suitable neighbors $K_{i j_{1}}^{ \pm}, K_{i j_{2}}^{ \pm}$and $K_{i j_{3}}^{ \pm}$, is significantly more difficult than the $2 \mathrm{D}$ algorithm. Below is a brief overview of the algorithm for backward neighbors (see figure 3). Therefore what one has to do is:

Step 1. sort the elements $K_{k}$ in the $\mathcal{W}(i)$ neighborhood in decreasing order of the value $\cos \left(\boldsymbol{B}_{k} \boldsymbol{B}_{i}, \boldsymbol{B}_{i} \boldsymbol{M}_{i j}\right)$, so as to establish an ordered list $\left(K_{1}, K_{2}, K_{3}, \ldots, K_{z}\right)$ where $z$ is the number of elements in $\mathcal{W}(i)$.

Step 2. determine the equation of the plane $\mathcal{P}_{1}$, which contains the centroid $\boldsymbol{B}_{1}$ of the first element $K_{1}$ in the sorted list, and with $\boldsymbol{B}_{i} \boldsymbol{M}_{i j}$ as normal vector.

Step 3. build a local orthonormate coordinate system $\left(\boldsymbol{O}_{1}, \boldsymbol{u}, \boldsymbol{v}\right)$ in the plane $\mathcal{P}_{1}$, where $\boldsymbol{O}_{1}$ is the intersection point between $\left(\boldsymbol{B}_{i} \boldsymbol{M}_{i j}\right)$ and $\mathcal{P}_{1}$. The basis vectors are defined as

$$
\boldsymbol{u}=\frac{\boldsymbol{O}_{1} \boldsymbol{B}_{1}}{\left\|\boldsymbol{O}_{1} \boldsymbol{B}_{1}\right\|},
$$

and $\boldsymbol{v}$ is any of the two unit vectors belonging to $\mathcal{P}_{1}$ and orthogonal to $\boldsymbol{u}$. Note that there might be a singularity when $\boldsymbol{B}_{1}$ is strictly located on the axis $\left(\boldsymbol{B}_{i} \boldsymbol{M}_{i j}\right)$, which means that $\boldsymbol{O}_{1}=\boldsymbol{B}_{1}$. This is for instance always the case for structured grids. This is not a problem, as in that case we thereby already have a consistent value on the axis $\left(\boldsymbol{B}_{i} \boldsymbol{M}_{i j}\right)$, and therefore do not need any further neighbor.

Step 4. determine $\boldsymbol{H}_{2}, \boldsymbol{H}_{3}$, the orthogonal projections of the centroids $\boldsymbol{B}_{2}$ and $\boldsymbol{B}_{3}$ (second and third elements of the sorted list) onto the plane $\mathcal{P}_{1}$ (see figure 3 ).

Step 5. pre-check the compatibility of the plane $\left(\boldsymbol{B}_{1} \boldsymbol{B}_{2} \boldsymbol{B}_{3}\right)$ with the required criteria, thanks to some geometrical tests in the plane $\mathcal{P}_{1}$. The first and compulsory criterion is that the intersection point between $\left(\boldsymbol{B}_{i} \boldsymbol{M}_{i j}\right)$ and the plane $\left(\boldsymbol{B}_{1} \boldsymbol{B}_{2} \boldsymbol{B}_{3}\right)$ 


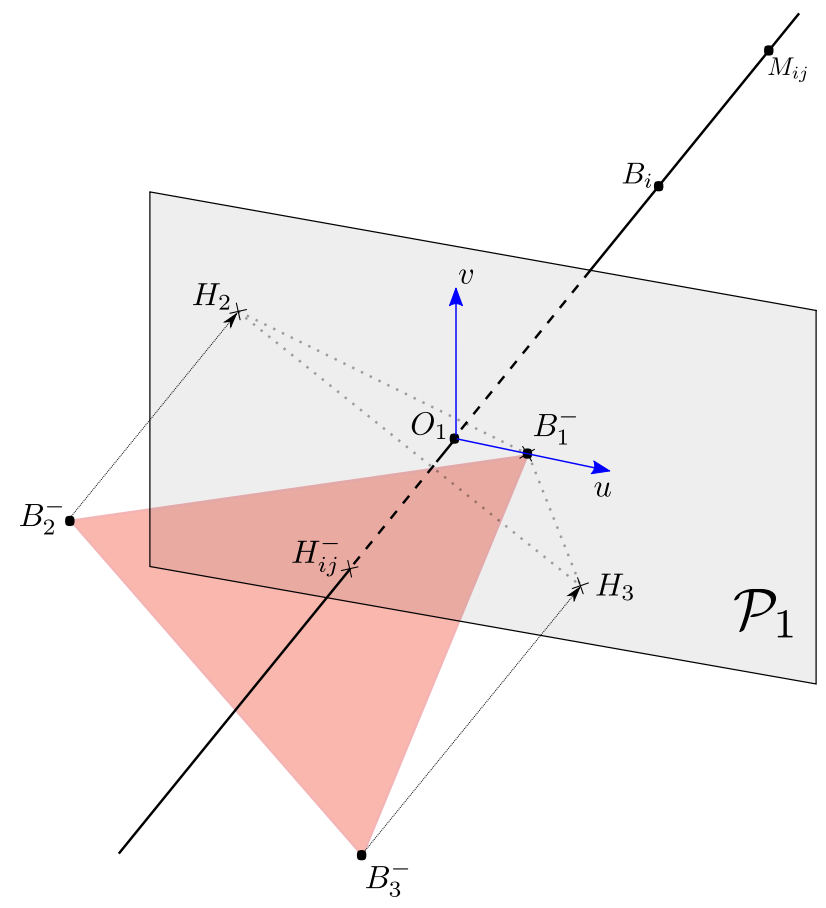

Figure 3: Illustration of the 3D algorithm to get the backward point $\boldsymbol{H}_{i j}^{-}$.

has to exist and furthermore has to be inside the triangle $\boldsymbol{B}_{1} \boldsymbol{B}_{2} \boldsymbol{B}_{3}$. But there are other optional criteria, for instance to prevent angles with too much obtuseness or acuteness.

Step 6. if OK, then elements $K_{1}, K_{2}$ and $K_{3}$ are assigned to $K_{i j_{1}}^{-}, K_{i j_{2}}^{-}$and $K_{i j_{3}}^{-}$. The intersection point is assigned to $\boldsymbol{H}_{i j}^{-}$, so nonnegative normalized barycentric coordinates $\beta_{i j_{1}}^{-}, \beta_{i j_{2}}^{-}, \beta_{i j_{3}}^{-}$can be computed, and therefore second-order consistent value $U_{H_{i j}^{-}}$as well.

Step 7. if not OK, and while not OK, then try again with another triplet of elements in the sorted list, namely $\left(\boldsymbol{B}_{\alpha} \boldsymbol{B}_{\beta} \boldsymbol{B}_{\gamma}\right)$ where the subscripts $\alpha, \beta, \gamma$ are successively incremented so as to minimize their sum. This means trying with $\left(\boldsymbol{B}_{1} \boldsymbol{B}_{2} \boldsymbol{B}_{4}\right)$, $\left(\boldsymbol{B}_{1} \boldsymbol{B}_{3} \boldsymbol{B}_{4}\right),\left(\boldsymbol{B}_{1} \boldsymbol{B}_{2} \boldsymbol{B}_{5}\right),\left(\boldsymbol{B}_{2} \boldsymbol{B}_{3} \boldsymbol{B}_{4}\right)$, and so on. This way, the approximation error when computing $U_{H_{i j}^{-}}$is also minimized. If no suitable plane has been found with combinations of backward elements, i.e. for which $\cos \left(\boldsymbol{B}_{k} \boldsymbol{B}_{i}, \boldsymbol{B}_{i} \boldsymbol{M}_{i j}\right)>$ 0 , then there is no reconstructed value and the scheme locally degenerates to first-order. But this only happens when an insufficient amount of neighbors is available, namely close to boundaries.

The procedure is similar to determine the forward neighbors, but starting from the last element of the sorted list (the most forward element in the neighborhood). It is also important to note that this algorithm only deals with geometrical data. As a result, it only has to be executed once. Therefore the data is stored in memory once and for all for a given mesh, and then used for every time step in the simulation process. 


\section{Properties of the reconstruction procedure}

\subsection{Second-order accuracy}

4.1.1. Accuracy of the fluxes approximation

The error made in the approximation of the flux is

$$
E=\int_{S_{i j}} \boldsymbol{F} \cdot \boldsymbol{n}_{i j} d S \quad-\quad \phi_{i j}\left(U_{i j}, U_{j i}, \boldsymbol{n}_{i j}\right)\left|S_{i j}\right| .
$$

$$
\text { flux }
$$

$$
\phi_{i j}\left(U_{i j}, U_{j i}, \boldsymbol{n}_{i j}\right)=\max \left(\boldsymbol{\lambda} \cdot \boldsymbol{n}_{i j}, 0\right) U_{i j}+\min \left(\boldsymbol{\lambda} \cdot \boldsymbol{n}_{i j}, 0\right) U_{j i},
$$

and assuming for instance $\boldsymbol{\lambda} \cdot \boldsymbol{n}_{i j} \geq 0$, then

$$
E=\boldsymbol{\lambda} \cdot \boldsymbol{n}_{i j}\left(\int_{S_{i j}} U(\boldsymbol{X}) d \boldsymbol{X}-U_{i j}\left|S_{i j}\right|\right) .
$$

Let $H(U)$ be the hessian matrix, then a Taylor expansion gives

$$
U(\boldsymbol{X})=U\left(\boldsymbol{M}_{i j}\right)+\left.\nabla U\right|_{M_{i j}} \cdot \boldsymbol{M}_{i j} \boldsymbol{X}+\left.\frac{1}{2} \boldsymbol{M}_{i j} \boldsymbol{X}^{t} H(U)\right|_{M_{i j}} \cdot \boldsymbol{M}_{i j} \boldsymbol{X}+o\left(\left\|\boldsymbol{M}_{i j} \boldsymbol{X}\right\|^{2}\right) .
$$

Now, the reconstruction will be second-order accurate if the error vanishes for linear

functions, that is for functions such that the Taylor expansion is reduced to

$$
U(\boldsymbol{X})=U\left(\boldsymbol{M}_{i j}\right)+\nabla U \cdot \boldsymbol{M}_{i j} \boldsymbol{X},
$$

where $\nabla U$ denotes the uniform gradient of $U$. Therefore, if we assume a linear function, then the normalized error $\bar{E}=E /\left(\left|S_{i j}\right| \boldsymbol{\lambda} \cdot \boldsymbol{n}_{i j}\right)$ reads in

$$
\bar{E}=\left[U\left(\boldsymbol{M}_{i j}\right)-U_{i j}\right]+\frac{1}{\left|S_{i j}\right|} \nabla U \cdot \int_{S_{i j}} \boldsymbol{M}_{i j} \boldsymbol{X} d \boldsymbol{X} .
$$

The second term on the right-hand side vanishes since the point $\boldsymbol{M}_{i j}$ is the barycentre of the face $S_{i j}$. Then, in order to get the second-order accuracy of the fluxes approximation, we need to prove that the reconstruction process yields $U_{i j}=U\left(\boldsymbol{M}_{i j}\right)$ for linear functions.

\subsubsection{Accuracy of the reconstructed values}

We first state the following result proved in Appendix A: due to the use of the barycentric coordinates, the values $U_{H_{i j}^{+}}$and $U_{H_{i j}^{-}}$are second-order approximations at points $\boldsymbol{H}_{i j}^{+}$and $\boldsymbol{H}_{i j}^{-}$, for both $2 \mathrm{D}$ and $3 \mathrm{D}$ configurations. Since we assume a linear function, it means that

$$
U_{H_{i j}^{+}}=U\left(\boldsymbol{H}_{i j}^{+}\right), \quad U_{H_{i j}^{-}}=U\left(\boldsymbol{H}_{i j}^{-}\right)
$$

Furthermore we can write for any $\boldsymbol{x} \in \Omega, \boldsymbol{x}_{0} \in \Omega$,

$$
U(\boldsymbol{x})=U\left(\boldsymbol{x}_{0}\right)+\nabla U \cdot\left(\boldsymbol{x}-\boldsymbol{x}_{0}\right),
$$


which leads to

$$
U_{H_{i j}^{+}}=U_{i}+\nabla U \cdot \boldsymbol{B}_{i} \boldsymbol{H}_{i j}^{+}, \quad U_{H_{i j}^{-}}=U_{i}+\nabla U \cdot \boldsymbol{B}_{i} \boldsymbol{H}_{i j}^{-} .
$$

Therefore, introducing the normalized vectors $\boldsymbol{v}_{i j}^{+}$and $\boldsymbol{v}_{i j}^{-}$, both belonging to the line $\left(\boldsymbol{B}_{i} \boldsymbol{M}_{i j}\right)$,

$$
\boldsymbol{v}_{i j}^{+}=\frac{\boldsymbol{B}_{i} \boldsymbol{H}_{i j}^{+}}{\left\|\boldsymbol{B}_{i} \boldsymbol{H}_{i j}^{+}\right\|}, \quad \boldsymbol{v}_{i j}^{-}=\frac{\boldsymbol{B}_{i} \boldsymbol{H}_{i j}^{-}}{\left\|\boldsymbol{B}_{i} \boldsymbol{H}_{i j}^{-}\right\|}, \quad \boldsymbol{v}_{i j}^{+}=-\boldsymbol{v}_{i j}^{-},
$$

the forward and backward slopes read in

$$
p_{i j}^{+}=\frac{U_{H_{i j}^{+}}-U_{i}}{\left\|\boldsymbol{B}_{i} \boldsymbol{H}_{i j}^{+}\right\|}=\nabla U \cdot \boldsymbol{v}_{i j}^{+}, \quad p_{i j}^{-}=\frac{U_{i}-U_{H_{i j}^{-}}}{\left\|\boldsymbol{B}_{i} \boldsymbol{H}_{i j}^{-}\right\|}=-\nabla U \cdot \boldsymbol{v}_{i j}^{-}=p_{i j}^{+} .
$$

Consequently, we have $r_{i j}=p_{i j}^{-} / p_{i j}^{+}=1$ for linear solutions. Finally, since any appropriate limiter exhibits $\varphi(1)=1$, then the reconstruted value $U_{i j}$ becomes

$$
\begin{gathered}
U_{i j}=U_{i}+p_{i j}^{+} \varphi\left(r_{i j}, \mathcal{G}_{i j}\right)\left\|\boldsymbol{B}_{i} \boldsymbol{M}_{i j}\right\|=U_{i}+p_{i j}^{+}\left\|\boldsymbol{B}_{i} \boldsymbol{M}_{i j}\right\|, \\
U_{i j}=U_{i}+\nabla U \cdot \boldsymbol{v}_{i j}^{+}\left\|\boldsymbol{B}_{i} \boldsymbol{M}_{i j}\right\|=U_{i}+\nabla U \cdot \boldsymbol{B}_{i} \boldsymbol{M}_{i j}=U\left(\boldsymbol{M}_{i j}\right),
\end{gathered}
$$

which completes the proof.

\section{2. $L^{\infty}$ stability}

We show in this section that the finite volume scheme (9) equipped with the generalized multislope reconstruction procedure can be written as a positive scheme with bounded coefficients, thereby implying a Discrete Maximum Principle and subsequently the $\mathrm{L}^{\infty}$-stability.

\subsubsection{Properties of the numerical flux and first rewriting of the scheme}

The numerical flux $\phi_{i j}$ at a given edge or face $S_{i j}$ is a function of two variables (if we drop the dependency on the normal vector $\boldsymbol{n}_{i j}$ ), denoted for instance $U_{1}$ and $U_{2}$. This means that

$$
\phi_{i j}=\phi_{i j}\left(U_{1}, U_{2}\right),
$$

where $U_{1}$ and $U_{2}$ are both assigned a certain evaluation of the scalar function coming from each side of $S_{i j}$. More specifically, $U_{1}$ and $U_{2}$ may be the cell-centered states $U_{i}^{n}$ and $U_{j}^{n}$, or they may be interpolated states $U_{i j}^{n}$ and $U_{j i}^{n}$, in which case the scheme is expected to upgrade from first-order to second-order accuracy.

Let us afterwards lay down some classical necessary properties of the numerical flux. Firstly, it has to be consistent with the physical flux. If we assume a case where the physical flux $\boldsymbol{F}$ depends only on $u$ and the scalar field has any uniform value $U$, then the condition of consistency of the numerical flux reads in

$$
\forall U \in \mathbb{R}, \phi_{i j}(U, U)=\boldsymbol{F}(U) \cdot \boldsymbol{n}_{i j},
$$

which implies the useful relation:

$$
\sum_{j \in \mathcal{V}(i)}\left|S_{i j}\right| \phi_{i j}(U, U)=\boldsymbol{F}(U) \cdot \sum_{j \in \mathcal{V}(i)}\left|S_{i j}\right| \boldsymbol{n}_{i j}=0 .
$$


Note that the condition of consistency is more complex when $\boldsymbol{F}$ depends also on $\boldsymbol{x}$ and $t$. In particular, the useful relation (39) is only approximate in this case as explained in [17]. For the sake of the demonstration, we therefore need to assume a physical flux only depending on $u$. Secondly, the numerical flux has to be monotonous, in the sense that

$$
\frac{\partial \phi_{i j}\left(U_{1}, U_{2}\right)}{\partial U_{1}} \geq 0, \quad \frac{\partial \phi_{i j}\left(U_{1}, U_{2}\right)}{\partial U_{2}} \leq 0
$$

According to relation (39), we can rewrite the scheme (9) as

$$
U_{i}^{n+1}=U_{i}^{n}-\sum_{j \in \mathcal{V}(i)} \Delta t \frac{\left|S_{i j}\right|}{\left|K_{i}\right|}\left[\phi_{i j}\left(U_{i j}, U_{j i}\right)-\phi_{i j}\left(U_{i j}, U_{i}\right)+\phi_{i j}\left(U_{i j}, U_{i}\right)-\phi_{i j}\left(U_{i}, U_{i}\right)\right] .
$$

The mean value theorem provides the following relations:

$\exists \widetilde{U_{i j}} \in\left[\min \left(U_{i}, U_{i j}\right), \max \left(U_{i}, U_{i j}\right)\right], \exists U_{i j}^{\star} \in\left[\min \left(U_{i}, U_{j i}\right), \max \left(U_{i}, U_{j i}\right)\right]$, such that:

$$
\begin{aligned}
& \frac{\partial \phi_{i j}}{\partial U_{1}}\left(\widetilde{U_{i j}}, U_{i}\right)=\frac{\phi_{i j}\left(U_{i j}, U_{i}\right)-\phi_{i j}\left(U_{i}, U_{i}\right)}{U_{i j}-U_{i}}, \\
& \frac{\partial \phi_{i j}}{\partial U_{2}}\left(U_{i j}, U_{i j}^{\star}\right)=\frac{\phi_{i j}\left(U_{i j}, U_{j i}\right)-\phi_{i j}\left(U_{i j}, U_{i}\right)}{U_{j i}-U_{i}},
\end{aligned}
$$

which leads to

$$
U_{i}^{n+1}=U_{i}^{n}-\sum_{j \in \mathcal{V}(i)} \Delta t \frac{\left|S_{i j}\right|}{\left|K_{i}\right|}\left[\frac{\partial \phi_{i j}}{\partial U_{1}}\left(\widetilde{U_{i j}}, U_{i}\right)\left(U_{i j}-U_{i}\right)+\frac{\partial \phi_{i j}}{\partial U_{2}}\left(U_{i j}, U_{i j}^{\star}\right)\left(U_{j i}-U_{i}\right)\right] .
$$

Next, we introduce the parameters below, which are nonnegative due to the monotonicity of the numerical flux,

$$
\nu_{i j}^{+}=\Delta t \frac{\left|S_{i j}\right|}{\left|K_{i}\right|} \frac{\partial \phi_{i j}}{\partial U_{1}}\left(\widetilde{U_{i j}}, U_{i}\right) \geq 0, \quad \nu_{i j}^{-}=-\Delta t \frac{\left|S_{i j}\right|}{\left|K_{i}\right|} \frac{\partial \phi_{i j}}{\partial U_{2}}\left(U_{i j}, U_{i j}^{\star}\right) \geq 0 .
$$

So we finally have the scheme under the form:

$$
U_{i}^{n+1}=U_{i}^{n}+\sum_{j \in \mathcal{V}(i)}\left[\nu_{i j}^{-}\left(U_{j i}-U_{i}\right)-\nu_{i j}^{+}\left(U_{i j}-U_{i}\right)\right]
$$

From this point forward, the objective is to rewrite the differences $U_{i j}-U_{i}$ and $U_{j i}-U_{i}$ so that we can write the scheme under the form:

$$
U_{i}^{n+1}=U_{i}^{n}+\sum_{j \in \mathcal{W}(i)} c_{i j}\left(U_{j}-U_{i}\right), \quad \text { with } \forall j, c_{i j} \geq 0 \quad \text { and } \quad \sum_{j \in \mathcal{W}(i)} c_{i j} \leq 1 .
$$

Note that we expect a sum on the $\mathcal{W}(i)$ neighborhood instead of the $\mathcal{V}(i)$ neighborhood, since the reconstruction procedure involves elements belonging to $\mathcal{W}(i)$. 
4.2.2. Focus on the term $U_{i j}-U_{i}$

As explained in [17], the classical strategy to get a nonnegative coefficient for this term consists in achieving an inversion sign property as follows:

$$
\begin{gathered}
U_{i j}-U_{i}=\sum_{k \in \mathcal{W}(i)} \omega_{i j k}\left(U_{i}-U_{k}\right), \\
\forall K_{i} \in \Omega, \forall j \in \mathcal{V}(i), \forall k \in \mathcal{W}(i), \quad \omega_{i j k} \geq 0 \quad \text { and } \sum_{k \in \mathcal{W}(i)} \omega_{i j k} \leq C_{\infty},
\end{gathered}
$$

where $C_{\infty}$ is a uniform constant. To do so, let us rewrite this term using the different relations given in the previous sections. We first introduce the geometric parameters

$$
\eta_{i j}^{-}=\frac{\left\|\boldsymbol{B}_{i} \boldsymbol{H}_{i j}^{-}\right\|}{\left\|\boldsymbol{B}_{i} \boldsymbol{M}_{i j}\right\|}, \quad \eta_{i j}^{+}=\frac{\left\|\boldsymbol{B}_{i} \boldsymbol{H}_{i j}^{+}\right\|}{\left\|\boldsymbol{B}_{i} \boldsymbol{M}_{i j}\right\|},
$$

305

we write $\varphi_{i j}=\varphi\left(r_{i j}, \mathcal{G}_{i j}\right)$, and using equations (11), (13), it comes

$$
U_{i j}-U_{i}=\left\|\boldsymbol{B}_{i} \boldsymbol{M}_{i j}\right\| p_{i j}^{+} \varphi_{i j}=\left\|\boldsymbol{B}_{i} \boldsymbol{M}_{i j}\right\| \frac{p_{i j}^{-}}{r_{i j}} \varphi_{i j}=\frac{\varphi_{i j}}{\eta_{i j}^{-} r_{i j}}\left(U_{i}-U_{H_{i j}^{-}}\right) .
$$

According to the equations (17) to (22), we can write for both the $2 \mathrm{D}$ and the $3 \mathrm{D}$ cases, with $d=2$ or $d=3$ respectively,

$$
U_{H_{i j}^{-}}=\sum_{l=1}^{d} \beta_{i j_{l}}^{-} U_{i j_{l}}^{-}, \quad \sum_{l=1}^{d} \beta_{i j_{l}}^{-}=1,
$$

where $U_{i j_{l}}^{-}$is the value in the mesh element $K_{i j_{l}}^{-}$which belongs to $\mathcal{W}(i)$. Therefore:

$$
U_{i j}-U_{i}=\frac{\varphi_{i j}}{\eta_{i j}^{-} r_{i j}}\left[U_{i} \sum_{l=1}^{d} \beta_{i j_{l}}^{-}-\sum_{l=1}^{d} \beta_{i j_{l}}^{-} U_{i j_{l}}^{-}\right]=\frac{\varphi_{i j}}{\eta_{i j}^{-} r_{i j}} \sum_{l=1}^{d} \beta_{i j_{l}}^{-}\left(U_{i}-U_{i j_{l}}^{-}\right) .
$$

Finally, if we introduce the notation

$$
\mathcal{L}_{i j}^{-}=\left\{K_{i j_{l}}^{-} \mid l \in \mathbb{N}, 1 \leq l \leq d\right\},
$$

to denote the set of selected backward neighboring elements, then we come up with

$$
U_{i j}-U_{i}=\sum_{k \in \mathcal{W}(i)} \omega_{i j k}\left(U_{i}-U_{k}\right),
$$

${ }_{310}$ with $\omega_{i j k}=\frac{\varphi_{i j}}{\eta_{i j}^{-} r_{i j}} \beta_{i j_{l}}^{-}$if $K_{k} \in \mathcal{L}_{i j}^{-}$, and $\omega_{i j k}=0$ if $K_{k} \notin \mathcal{L}_{i j}^{-}$. So we do have the nonnegativity of the $\omega_{i j k}$ if the limiter is such that $\varphi_{i j}=0$ for $r_{i j} \leq 0$, and $\varphi_{i j} \geq 0$ when $r_{i j}>0$. Besides, the sum reads in

$$
\sum_{k \in \mathcal{W}(i)} \omega_{i j k}=\frac{\varphi_{i j}}{\eta_{i j}^{-} r_{i j}},
$$

which will be uniformly bounded with an ad hoc limiter. 


\subsubsection{Focus on the term $U_{j i}-U_{i}$}

$315 \quad$ According to relation (12), we have

$$
U_{j i}=U_{j}+\left\|\boldsymbol{B}_{j} \boldsymbol{M}_{i j}\right\| p_{j i}^{+} \varphi_{j i} .
$$

Then:

$$
\begin{gathered}
U_{j i}-U_{i}=U_{j}-U_{i}+\frac{\left\|\boldsymbol{B}_{j} \boldsymbol{M}_{i j}\right\|}{\left\|\boldsymbol{B}_{j} \boldsymbol{H}_{j i}^{+}\right\|} \varphi_{j i}\left(U_{H_{j i}}^{+}-U_{j}\right), \\
U_{j i}-U_{i}=\left(U_{j}-U_{i}\right)\left(1-\frac{\varphi_{j i}}{\eta_{j i}^{+}}\right)+\left(U_{H_{j i}}^{+}-U_{i}\right) \frac{\varphi_{j i}}{\eta_{j i}^{+}} .
\end{gathered}
$$

As previously for the $U_{i j}-U_{i}$ term, using the definition of $U_{H_{j i}}^{+}$, we can write

$$
U_{H_{j i}}^{+}-U_{i}=\sum_{l=1}^{d} \beta_{j i_{l}}^{+}\left(U_{j i_{l}}^{+}-U_{i}\right)
$$

where $U_{j i_{l}}^{+}$is the value in the mesh element $K_{j i_{l}}^{+}$, which belongs to $\mathcal{W}(j)$ by construction. 320 Then, for the sake of the demonstration, we need to assume that the following property holds:

Hypothesis 1. The elements $K_{j i_{l}}^{+}$chosen in order to compute the value $U_{H_{j i}}^{+}$, which belong to the $\mathcal{W}(j)$ neighborhood by construction, also belong to the $\mathcal{W}(i)$ neighborhood.

Note that most meshes will naturally exhibit this property (see figure 1 as an illustration). If not, then it can still be enforced when the geometric algorithm is executed to find the forward neigbors. Now with this property, and introducing the notation

$$
\mathcal{L}_{j i}^{+}=\left\{K_{j i_{l}}^{+} \mid l \in \mathbb{N}, 1 \leq l \leq d\right\},
$$

to denote the set of selected forward neighboring elements, we therefore get

$$
\left(U_{H_{j i}}^{+}-U_{i}\right) \frac{\varphi_{j i}}{\eta_{j i}^{+}}=\sum_{k \in \mathcal{W}(i)} \lambda_{i j k}\left(U_{k}-U_{i}\right),
$$

with $\lambda_{i j k}=\frac{\varphi_{j i}}{\eta_{j i}^{+}} \beta_{j i_{l}}^{+}$if $K_{k} \in \mathcal{L}_{j i}^{+}$, and $\lambda_{i j k}=0$ if $K_{k} \notin \mathcal{L}_{j i}^{+}$. So, once again we do have the nonnegativity of the $\lambda_{i j k}$ if the limiter is nonnegative. Besides, the sum reads in

$$
\sum_{k \in \mathcal{W}(i)} \lambda_{i j k}=\frac{\varphi_{j i}}{\eta_{j i}^{+}}
$$

which, again, will be uniformly bounded with an ad hoc limiter. We finally get for the $U_{j i}-U_{i}$ term:

$$
U_{j i}-U_{i}=\left(U_{j}-U_{i}\right)\left(1-\frac{\varphi_{j i}}{\eta_{j i}^{+}}\right)+\sum_{k \in \mathcal{W}(i)} \lambda_{i j k}\left(U_{k}-U_{i}\right) .
$$




\subsubsection{Second rewriting of the scheme}

Now, if we introduce in (46) the relations found for $U_{i j}-U_{i}$ and $U_{j i}-U_{i}$, then the scheme reads in

$$
\begin{aligned}
U_{i}^{n+1}=U_{i}^{n}+\sum_{j \in \mathcal{V}(i)}\left[\nu_{i j}^{-}\left(1-\frac{\varphi_{j i}}{\eta_{j i}^{+}}\right)\left(U_{j}-U_{i}\right)\right. & +\nu_{i j}^{-} \sum_{k \in \mathcal{W}(i)} \lambda_{i j k}\left(U_{k}-U_{i}\right) \\
& \left.-\nu_{i j}^{+} \sum_{k \in \mathcal{W}(i)} \omega_{i j k}\left(U_{i}-U_{k}\right)\right] .
\end{aligned}
$$

Afterwards, imposing $\nu_{i j}^{-}=0$ if $j \in \mathcal{W}(i), j \notin \mathcal{V}(i)$, allows to extend the sum on $j$ over the set $\mathcal{W}(i)$ for the first term of the sum in relation (64). For the last two terms of the sum on $j$, we can permute the sums on $j$ and $k$. In the case of the second term for example, this means:

$$
\begin{aligned}
\sum_{j \in \mathcal{V}(i)} \nu_{i j}^{-} \sum_{k \in \mathcal{W}(i)} \lambda_{i j k}\left(U_{k}-U_{i}\right) & =\sum_{k \in \mathcal{W}(i)}\left(U_{k}-U_{i}\right)\left(\sum_{j \in \mathcal{V}(i)} \lambda_{i j k} \nu_{i j}^{-}\right) \\
& =\sum_{j \in \mathcal{W}(i)}\left(U_{j}-U_{i}\right)\left(\sum_{k \in \mathcal{V}(i)} \lambda_{i k j} \nu_{i k}^{-}\right)
\end{aligned}
$$

A similar result holds for the third term, so that the relation (64) becomes

$$
U_{i}^{n+1}=U_{i}^{n}+\sum_{j \in \mathcal{W}(i)}\left(U_{j}-U_{i}\right)\left[\nu_{i j}^{-}\left(1-\frac{\varphi_{j i}}{\eta_{j i}^{+}}\right)+\sum_{k \in \mathcal{V}(i)}\left(\omega_{i k j} \nu_{i k}^{+}+\lambda_{i k j} \nu_{i k}^{-}\right)\right]
$$

Therefore the scheme can finally be written under the following form:

$$
U_{i}^{n+1}=U_{i}^{n}+\sum_{j \in \mathcal{W}(i)} c_{i j}\left(U_{j}-U_{i}\right)
$$

where the coefficients $c_{i j}$ read in:

$$
c_{i j}=\nu_{i j}^{-}\left(1-\frac{\varphi_{j i}}{\eta_{j i}^{+}}\right)+\sum_{k \in \mathcal{V}(i)}\left(\omega_{i k j} \nu_{i k}^{+}+\lambda_{i k j} \nu_{i k}^{-}\right)
$$

\subsubsection{Nonnegativity and boundedness of the coefficients}

As mentioned in relation (45), the coefficients $\nu_{i j}^{-}$and $\nu_{i j}^{+}$are nonnegative. Further340 more, if the limiter function is such that

$$
\forall K_{i} \in \Omega, \forall j \in \mathcal{V}(i), \varphi_{i j} \in\left[0, \eta_{i j}^{+}\right]
$$


then we get the nonnegativity of the coefficients $c_{i j}$. Let us now write their sum:

$$
\begin{aligned}
& \sum_{j \in \mathcal{W}(i)} c_{i j}=\sum_{j \in \mathcal{W}(i)} \nu_{i j}^{-}\left(1-\frac{\varphi_{j i}}{\eta_{j i}^{+}}\right)+\sum_{j \in \mathcal{W}(i)} \sum_{k \in \mathcal{V}(i)}\left(\omega_{i k j} \nu_{i k}^{+}+\lambda_{i k j} \nu_{i k}^{-}\right) \\
& \sum_{j \in \mathcal{W}(i)} c_{i j}=\sum_{j \in \mathcal{W}(i)} \nu_{i j}^{-}\left(1-\frac{\varphi_{j i}}{\eta_{j i}^{+}}\right)+\sum_{j \in \mathcal{V}(i)}\left[\nu_{i j}^{+}\left(\sum_{k \in \mathcal{W}(i)} \omega_{i j k}\right)+\nu_{i j}^{-}\left(\sum_{k \in \mathcal{W}(i)} \lambda_{i j k}\right)\right] .
\end{aligned}
$$

Using relations (55) and (62), and because $\nu_{i j}^{-}=0$ if $j \in \mathcal{W}(i), j \notin \mathcal{V}(i)$, we obtain

$$
\sum_{j \in \mathcal{W}(i)} c_{i j}=\sum_{j \in \mathcal{V}(i)} \nu_{i j}^{-}\left(1-\frac{\varphi_{j i}}{\eta_{j i}^{+}}\right)+\sum_{j \in \mathcal{V}(i)}\left(\nu_{i j}^{+} \frac{\varphi_{i j}}{\eta_{i j}^{-} r_{i j}}+\nu_{i j}^{-} \frac{\varphi_{j i}}{\eta_{j i}^{+}}\right) .
$$

And the sum finally reads in

$$
\sum_{j \in \mathcal{W}(i)} c_{i j}=\sum_{j \in \mathcal{V}(i)}\left(\nu_{i j}^{-}+\nu_{i j}^{+} \frac{\varphi_{i j}}{\eta_{i j}^{-} r_{i j}}\right) .
$$

Now we know that stability is conditioned by $\sum c_{i j} \leq 1$, since the value $U_{i}^{n+1}$ is thereby obtained as a convex combination between values within the $\mathcal{W}(i)$ neighborhood. This indeed complies with the DMP stated in (10) just by considering the $\mathcal{W}$-like neighborhood as the set $\mathcal{U}$ used in Definition 1. Introducing $N_{i}=\operatorname{card}[\mathcal{V}(i)]$, i.e. the number of edges or faces of the element $K_{i}$, then from relation (73) we can derive two sufficient stability conditions:

$$
\forall K_{i} \in \Omega, \forall j \in \mathcal{V}(i), \text { if } \nu_{i j}^{-} \leq \frac{1}{2 N_{i}} \text {, and if } \nu_{i j}^{+} \frac{\varphi_{i j}}{\eta_{i j}^{-} r_{i j}} \leq \frac{1}{2 N_{i}}, \text { then } \sum_{j \in \mathcal{V}(i)} c_{i j} \leq 1 .
$$

Let us now introduce the parameters

$$
N=\max _{K_{i} \in \Omega} N_{i}, h_{0}=\min _{\substack{K_{i} \in \Omega \\ j \in \mathcal{V}(i)}} \frac{\left|K_{i}\right|}{\left|S_{i j}\right|}, \quad M=\max _{\substack{K_{i} \in \Omega \\ j \in \mathcal{V}(i) \\ t \in[0, T]}}\left[\frac{\partial \phi_{i j}}{\partial U_{1}}\left(\widetilde{U_{i j}}, U_{i}\right),-\frac{\partial \phi_{i j}}{\partial U_{2}}\left(U_{i j}, U_{i j}^{\star}\right)\right],
$$

and let $\nu_{\max }=\Delta t M / h_{0}$ denote the maximum reachable CFL number, such that

$$
\forall K_{i} \in \Omega, \quad \forall j \in \mathcal{V}(i), \quad \forall t \in[0, T], \quad \nu_{i j}^{-} \leq \nu_{\max }, \quad \nu_{i j}^{+} \leq \nu_{\max } .
$$

Then the first condition in relation (74) is included in the uniform sufficient condition:

$$
\nu_{\max } \leq \frac{1}{2 N}
$$

which gives rise to a sufficient stability condition on the time step:

$$
\Delta t \leq \frac{h_{0}}{2 M N} .
$$


The second sufficient condition in relation (74) leads to a condition on the limiter:

$$
\varphi_{i j} \leq \frac{\eta_{i j}^{-} r_{i j}}{2 N_{i} \nu_{i j}^{+}}
$$

We can keep this condition as is, which will generate a CFL-dependent limiter. Or, using the fact that $2 N_{i} \nu_{i j}^{+} \leq 1$, we can derive another sufficient condition:

$$
\varphi_{i j} \leq \eta_{i j}^{-} r_{i j}
$$

which will generate a more classical CFL-independent limiter. Note that condition (79) is less restrictive than condition (80) which means that CFL-dependent limiters are expected to be less dissipative than their CFL-independent counterparts.

\subsubsection{Summary of the $L^{\infty}$ stability proof}

Finally, the results proved in section 4.2 are summarized in the proposition below.

Proposition 1. Under the assumptions made for the physical and numerical fluxes in section 4.2.1, and under the Hypothesis 1 concerning the mesh and the forward neighbors determination process, the finite volume MUSCL scheme (9) based on the generalized multislope reconstruction complies with the discrete maximum principle (DMP) stated in Definition 1, and therefore is $L^{\infty}$-stable, under the sufficient condition on the time step:

$$
\Delta t \leq \frac{h_{0}}{2 M N}
$$

and under the CFL-dependent sufficient condition on the limiter function:

$$
0 \leq \varphi_{i j} \leq \min \left(\eta_{i j}^{+}, \frac{\eta_{i j}^{-} r_{i j}}{2 N_{i} \nu_{i j}^{+}}\right) \text {, }
$$

in which is included a more classical CFL-independent sufficient condition on the limiter:

$$
0 \leq \varphi_{i j} \leq \min \left(\eta_{i j}^{+}, \eta_{i j}^{-} r_{i j}\right)
$$

Of course, these are only sufficient conditions, so larger time steps can be used in practice without making the scheme unstable. Similarly, one could envisage less restrictive bounding conditions for the limiters, for instance by inserting a coefficient $k>1$ in the upper bound as follows:

$$
0 \leq \varphi_{i j} \leq \min \left(\eta_{i j}^{+}, \frac{\eta_{i j}^{-} r_{i j} k}{2 N_{i} \nu_{i j}^{+}}\right)
$$

Depending on the case under study, a well-chosen $k>1$ is likely to improve the scheme accuracy while still preserving $\sum c_{i j} \leq 1$ and therefore the stability. However we will not push this issue further and will just consider $k=1$ in the sequel. Finally, keeping 
in mind that the second-order consistency proved in section 4.1 requires the following additional property of the limiter:

$$
\text { if } r_{i j}=1 \text { then } \varphi_{i j}=1,
$$

then we understand that the design of an overall optimal limiter relies on the best combination between the latter property (giving the optimal shape for smooth solutions, i.e. $r_{i j} \approx 1$ ), and the bounds of the stability region above-mentioned (giving the optimal shape for sharp bending solutions, i.e. $r_{i j} \ll 1$ and $\left.r_{i j} \gg 1\right)$. This is the purpose of the next section.

\section{Design of well-adapted limiters}

375

\subsubsection{CFL-Superbee limiter}

$$
\varphi_{\nu-S u p}\left(r_{i j}, \eta_{i j}^{+}, \eta_{i j}^{-}, \nu_{i j}^{+}, N_{i}\right)=\max \left[0, \min \left(\frac{\eta_{i j}^{-} r_{i j}}{2 N_{i} \nu_{i j}^{+}}, 1\right), \min \left(r_{i j}, \eta_{i j}^{+}\right)\right]
$$


This is the most performant limiter for sharp gradient regions, where $r_{i j} \ll 1$ or $r_{i j} \gg 1$, as it follows the upper bound of the stability region. Furthermore, it respects the linearity preserving property since $\varphi_{\nu \text {-Sup }}(1)=1$, but only weakly. Indeed, the switching between two different, overcompressive linear schemes around the point $r_{i j}=1$ brings about the well-known squaring effect usually observed with the classical Superbee limiter (see [27]). A CFL-independent version is obtained by setting $1 /\left(2 N_{i} \nu_{i j}^{+}\right)=1$, which results in the classical Superbee limiter for irregular grids.

\subsubsection{CFL-third order limiter}

$$
\varphi_{\nu-o 3}\left(r_{i j}, \eta_{i j}^{+}, \eta_{i j}^{-}, \nu_{i j}^{+}, N_{i}\right)=\max \left(0, \min \left[\varphi_{\nu-S u p}, 1+\frac{1+\nu_{i j}^{+}}{3}\left(r_{i j}-1\right)\right]\right)
$$

This limiter is actually the third-order scheme as long as $\varphi$ is included in the stability region, and is the upper bound of the latter otherwise (see figure 4). It is built upon an appropriate averaging between the Lax-Wendroff and the Beam-Warming schemes, the weight given to the latter increasing with the CFL number as pointed out in [26]. As a result, it exhibits excellent accuracy in the smooth gradient $\left(r_{i j} \approx 1\right)$ region.

Furthermore, for some particular values of the CFL number, it is equivalent to some classical CFL-independent limiters that include $\kappa$-schemes for $r_{i j} \approx 1$. For instance, if we consider the general class of the linear schemes written in the flux-limiter formalism as in [27]:

$$
\varphi_{\kappa}\left(r_{i j}\right)=\frac{1}{2}\left[(1+\kappa) r_{i j}+(1-\kappa)\right]
$$

then, in the $\left(r_{i j} \approx 1\right)$ region, $\varphi_{\nu-o 3}\left(\nu_{i j}^{+}=1\right)$ is equivalent to the $\kappa=\frac{1}{3}$ Cubic-Upwind linear scheme, on which is based the classical Koren limiter. Similarly, $\varphi_{\nu-o 3}\left(\nu_{i j}^{+}=\right.$ $0.5)$ is equivalent to the $\kappa=0$ Fromm's linear scheme, on which is based the classical Monotonized Central limiter.

\subsection{Generation of a hybrid CFL-dependent limiter}

We now introduce a way to generate a tailor-made limiter, from well-chosen pivot 425 limiters. The key point when designing an accurate limiter is to combine the performance of the most compressive limiters in the sharp bending zones, with the performance of the third-order scheme in the smooth region. For that purpose we use a parameterized switch function in a similar way as Harten in [7]. Dropping the subscripts ij for the sake of conciseness, and distinguishing between the intervals $r \in[0,1[$ and $r \in[1,+\infty[$, the switch function reads in

$$
h(r)=\left\{\begin{array}{ll}
{\left[1-\tanh \left(a r^{b}\right)\right](1-r)^{c}} & \text { if } 0<r<1 \\
\left(\tanh \left[d(r-1)^{e}\right]\right)^{f} & \text { if } r \geq 1
\end{array},\right.
$$

where tanh is the hyperbolic tangent function, and $a, b, c, d, e$ and $f$ are parameters to be chosen. We drop the dependency of the limiters on the geometric parameters, then 
a tailor-made limiter can be obtained according to the following convex combination between two chosen pivot limiters $\varphi_{1}$ and $\varphi_{2}$ :

$$
\varphi(r)=[1-h(r)] \varphi_{1}(r)+h(r) \varphi_{2}(r) .
$$

435 The pivot limiters are chosen among the CFL-dependent limiters introduced in section 5.1. We therefore assign the third-order limiter to the pivot limiter $\varphi_{1}(r)$ :

$$
\varphi_{1}=\varphi_{\nu-o 3}
$$

whereas we select the limiter which is tight-fitted to the upper bound of the stability region as the pivot limiter $\varphi_{2}(r)$ :

$$
\varphi_{2}=\varphi_{\nu-S u p}
$$

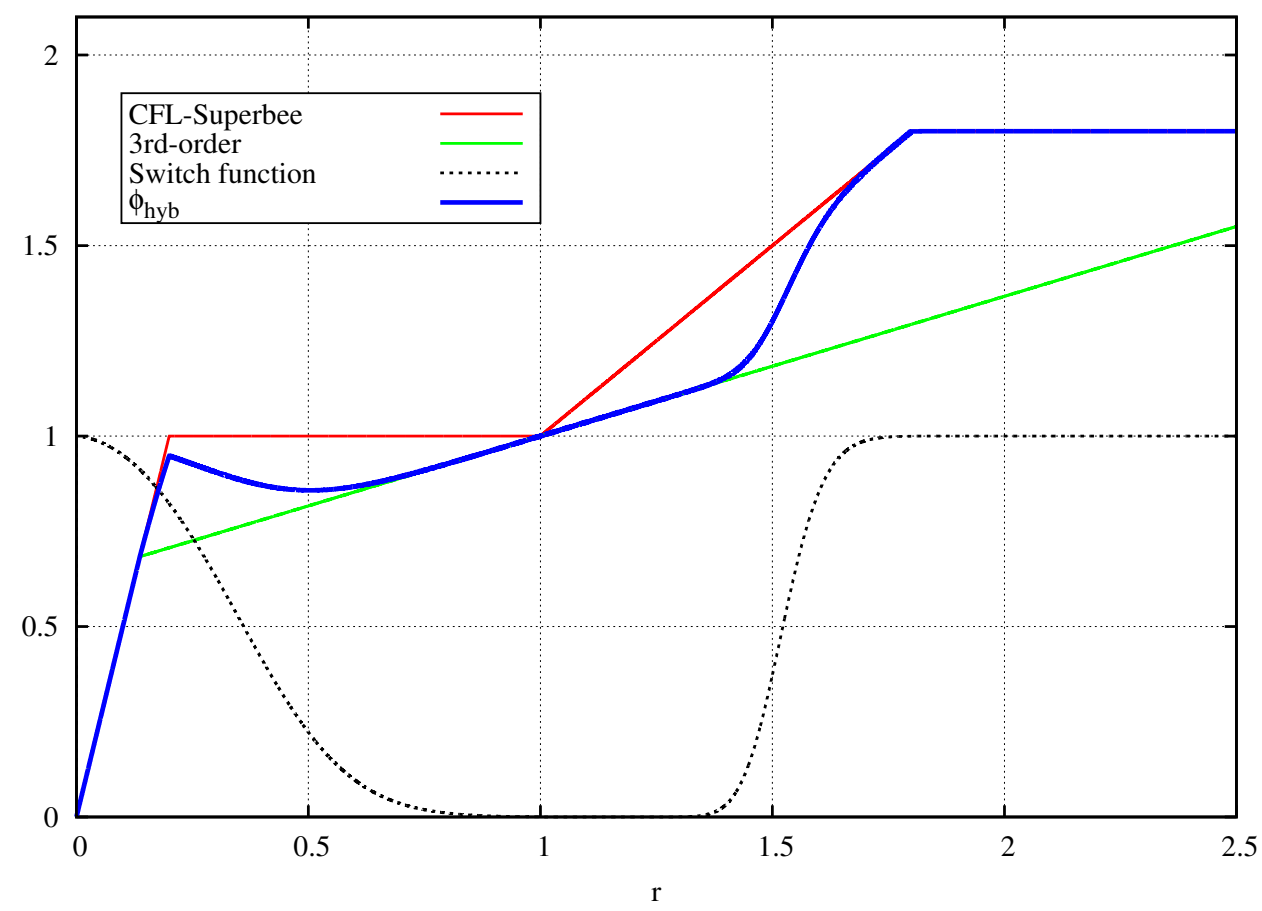

Figure 4: Switch function, pivots limiters, and hybrid limiter.

The final limiter lays between the two pivots limiters since $h(r)$ is constrained in the interval $[0,1]$ by definition. And this is the value of the parameters that determines the way the final limiter switches from one to the other. The attractiveness of such a strategy is that the slope of the final limiter is controled by that of $\varphi_{2}$ for $r \rightarrow 0^{+}$, while it is controled by that of $\varphi_{1}$ for $r \approx 1$ (see proof in Appendix B). Lastly, the values of the fitting parameters, which determine the shape of the hybrid limiter, are here chosen as 445 follows:

$$
a=4, \quad b=2, \quad c=0.1, \quad d=10, \quad e=3, \quad f=6 .
$$


Note that one could very well try to use different values, thus modifying the shape of the generated limiter. However the influence of these parameters will not be investigated in this paper, and we will stick to the above values in the sequel. An illustration of the hybrid limiter thus obtained is provided on figure 4, for randomly-chosen geometric 450 parameters, namely $\eta_{i j}^{-}=3, \eta_{i j}^{+}=1.8, \nu_{i j}^{+}=0.2, N_{i}=3$. The advantage of this limiting process over more classical limiters is illustrated by the numerical tests exposed in the next section.

\section{Numerical results}

\subsection{Dealing with boundary conditions}

Before going into detail about the numerical tests, some precision is brought here on the treatment made for boundary conditions. This issue concerns both the computation of the slopes in the multislope reconstruction and the computation of the numerical flux in the time-stepping process.

\subsubsection{Multislope reconstruction}

The way the multislope reconstruction is affected by boundaries is rather obvious. Indeed, the number of elements in the $\mathcal{W}(i)$ neighborhood is inevitably reduced when $K_{i}$ is close to a boundary. As a result, there might be cases where no suitable neighbors have been found to compute the backward and / or the forward slopes for a given face $S_{i j}$. When this happens, the slopes are just set to zero, and as a result we get back to 465 first-order upwinding for the face under consideration. This is the immediate and safe way to still comply with the stability requirements exposed in section 4.2.

Furthermore, the multislope procedure is easily and readily compatible with multidomain and parallel computing, as long as the required information is exchanged between domains. This can be achieved for instance using MPI libraries. More specifically, one

${ }_{470}$ needs to make sure that the elements sharing a vertex with the domain interface are exchanged, not only the elements sharing a face with it.

\subsubsection{Computation of the numerical fluxes and time-stepping process}

In a classical way, the case of boundary control volumes is treated using ghost cells. For any face $S_{i j}$ belonging to the boundary $\partial \Omega$, we create the ghost element $\widetilde{K}_{i}$ with 475 respect to $K_{i}$, on which the boundary condition is prescribed. As a result, the computation of the numerical flux on the face $S_{i j}$, and then the time-stepping process in the control volume $K_{i}$, can be performed in the same way as if $K_{i}$ were strictly inside the domain.

\section{2. $2 D$ rotation test case}

To evaluate the performance of the new reconstruction procedure, we consider the $2 \mathrm{D}$ rotation problem (94), also used for instance in [15].

$$
\left.\left.\partial_{t} u(x, y, t)+\boldsymbol{\lambda}(x, y) \cdot \boldsymbol{\nabla} u(x, y, t)=0 \quad(x, y) \in \Omega=[0,1] \times[0,1], \quad t \in\right] 0, T\right]
$$

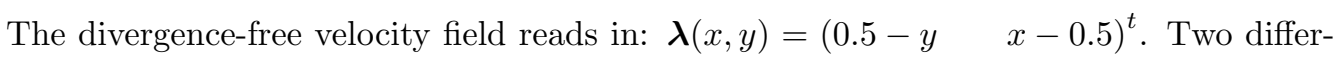
ent initial solutions $u_{0}(x, y)$ are considered, respectively a discontinuous and a smooth 
function (see figure 7), which are convected during one revolution, i.e. a simulation time of $T=2 \pi$. In order to assess the ability of the method to deal with completely general meshes, six kinds of mesh are used, namely cartesian, structured triangles, unstructured triangles, quads, or polygons. They range from $G_{1}$ to $G_{6}$ (see table 1 and figures 5 and 6). Successive computations with increasing number of elements $\mathcal{N}$ on each kind of mesh enables us to calculate the scheme error, and then draw comparisons. The numbers of 490 elements used for each grid are reported in table 2.

\begin{tabular}{l|cccccc}
\hline Grids & $G_{1}$ & $G_{2}$ & $G_{3}$ & $G_{4}$ & $G_{5}$ & $G_{6}$ \\
\hline Type & Cartesian & Tri "scottish" & Tri "diagonal" & Tri "free" & Quad & Poly \\
\hline
\end{tabular}

Table 1: List of the structured and unstructed grids used.

\begin{tabular}{c|llllll}
\hline Grids & $G_{1}$ & $G_{2}$ & $G_{3}$ & $G_{4}$ & $G_{5}$ & $G_{6}$ \\
\hline-- & - & - & - & 1758 & 1642 & 1871 \\
- & 1600 & 1600 & 3200 & 3558 & 3721 & 3670 \\
+ & 6400 & 6400 & 12800 & 7033 & 7180 & 7050 \\
++ & 25600 & 25600 & 51200 & 13720 & 14884 & 14404 \\
\hline
\end{tabular}

Table 2: Increasing number of elements $\mathcal{N}$. Grids $G_{1}$ to $G_{6}$.

We hereby want to evaluate the performance of the reconstruction method over a firstorder upwind scheme and over a classical monoslope approach, as well as the respective advantages of the limiting strategies that have been introduced. In the monoslope technique, a least squares formula is used to evaluate the gradient from the face neighborhood ${ }_{495} \mathcal{V}(i)$. Then a directional limitation procedure is applied to the gradient. The procedure is described in details in [28], and is inspired by the works of [25], [12] and [29]. The principle is to find the minimum and maximum values in the closest neighborhood, and to limit the gradient so that the maximum principle is respected. This monoslope method will be called "Limited Gradient" (LG) in the sequel.

In order to see the influence of whether or not introducing the CFL number in the limiters, we use an explicit RK2 time-stepping scheme, and we choose to perform all computations with the maximum Courant number $\nu_{\max } \approx 0.1$. To do so, and based on the definition of $\nu_{\max }$ in section 4.2.5, we need to adjust the time step for every mesh according to

$$
\Delta t=\nu_{\max } \frac{h}{M},
$$

505 where

$$
h \approx \sqrt{\frac{|\Omega|}{\mathcal{N}}}=\frac{1}{\sqrt{\mathcal{N}}}
$$

is the characteristic size of a given mesh (used here instead of the minimum size $h_{0}$ defined in section 4.2.5), and $|\Omega|=1$ is the surface area of the computational domain. Besides, $M$ denotes the maximum value of the norm of the velocity field, which here 
reads in

$$
M=\max _{(x, y) \in \Omega}\|\boldsymbol{\lambda}(x, y)\| \approx 0.707 .
$$

Let us introduce the error in the $L^{1}$ norm, defined as follows:

$$
\epsilon_{1}=\sum_{K_{i}}\left|K_{i}\right|\left|U_{i}(t=2 \pi)-U_{i}(t=0)\right| .
$$

where $U_{i}(t=2 \pi)$ is the discrete solution at the center of $K_{i}$ after one revolution, and $U_{i}(t=0)$ is the projection on the mesh of the initial function $u_{0}(x, y)$, which can be approximated as the exact solution at time $t=2 \pi$. We furthermore define the error in $L^{\infty}$ norm as

$$
\epsilon_{\infty}=\max _{K_{i}}\left|U_{i}(t=2 \pi)-U_{i}(t=0)\right| .
$$

515 The $L^{1}$ norm indicates the global diffusive error of the scheme, and as a result is interesting for both discontinuous and smooth cases. The $L^{\infty}$ norm is only relevant for smooth cases, and gives information about the loss of amplitude of the convected function. Finally, we can derive the average schemes order $\alpha$, either in the $L^{1}$ norm or in the $L^{\infty}$ norm. The scheme error in a given norm $n$ is supposed to verify $\epsilon_{n}=C_{n} h^{\alpha_{n}}$, where $C_{n}$

520 is a constant. Therefore, $\alpha_{n}$ is obtained by computing the slope of the linear regressions $\log \epsilon_{n}=\alpha_{n} \log h+\log C_{n}$, which are drawn on the various figures afterwards.

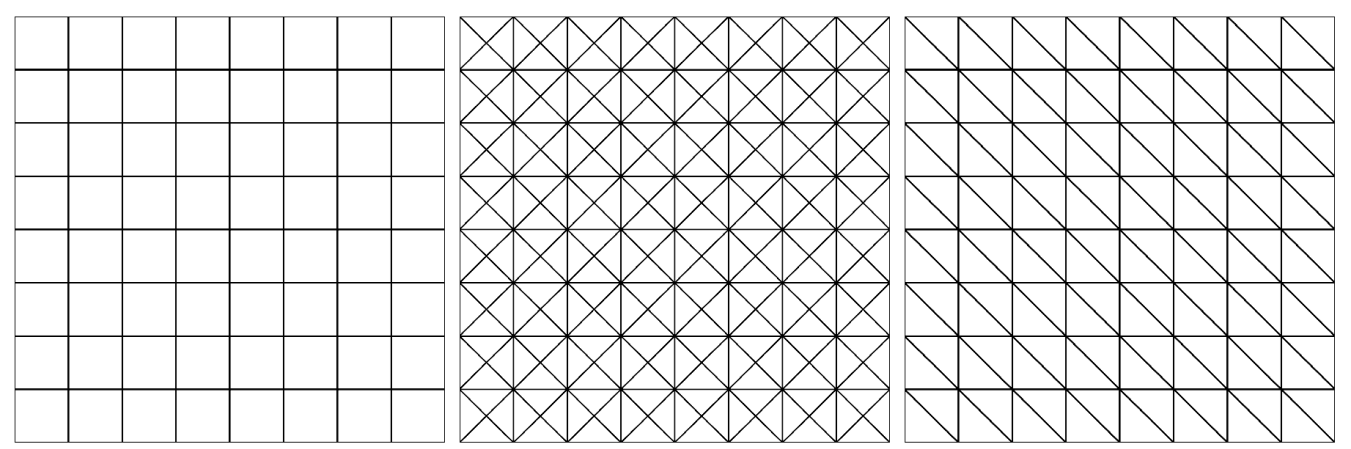

Figure 5: Typical grids $G_{1}, G_{2}$, and $G_{3}$.

\subsubsection{Discontinuous initial solution}

We use the following function as the discontinuous initial solution:

$$
u_{0}(x, y)=\left\{\begin{array}{ll}
1 & \text { if } \quad r \leq 0.15 \\
0 & \text { if } \quad r>0.15
\end{array} \quad \text { with } \quad r=\sqrt{(x-0.3)^{2}+(y-0.3)^{2}}\right.
$$

The results for the discontinuous case on the six grids are shown on figures 8,9 , and 10. We draw linear regressions of the error in $L^{1}$ norm versus the characteristic size $h$, for five different convective schemes: the First Order Upwind (denoted by FOU on the figures), the Limited Gradient method (LG), and the Generalized Multislope technique successively equipped with the classical CFL-independent Superbee limiter extended to 


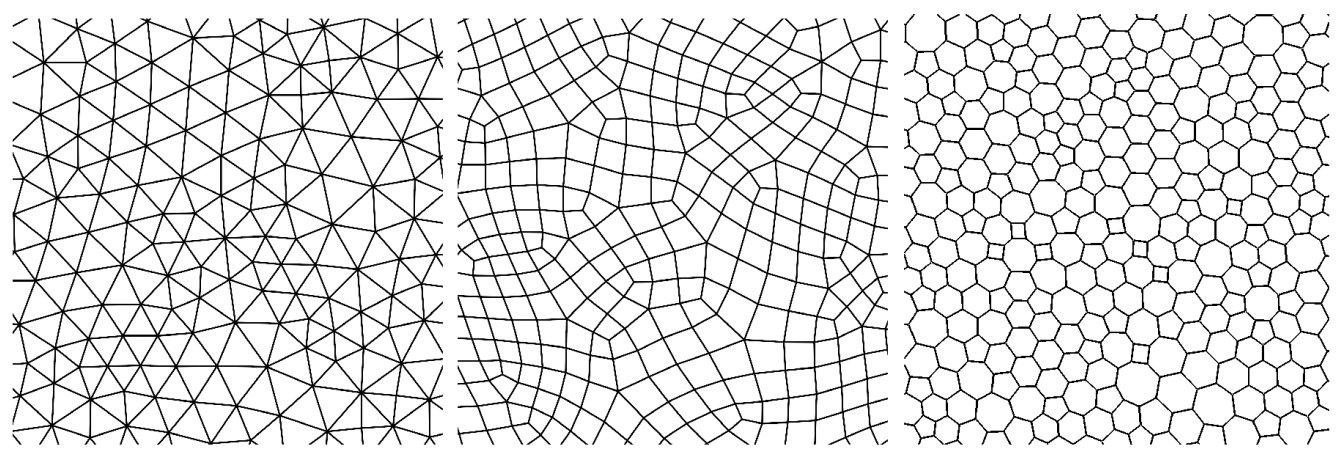

Figure 6: Typical grids $G_{4}, G_{5}$, and $G_{6}$.
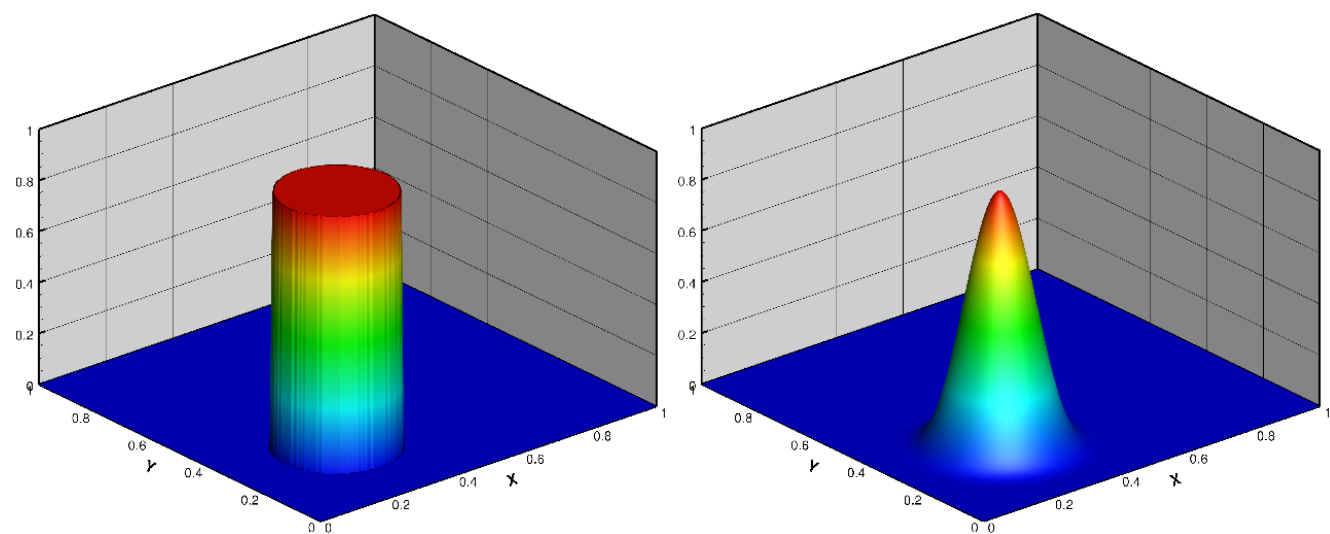

Figure 7: Discontinuous and continuous initial solutions in the coordinate system $(x, y, u)$.

irregular grids (GM-Sup), namely equation (86) with $2 N_{i} \nu_{i j}^{+}=1$, the CFL-Superbee 530 limiter introduced in (86) (GM-CFL-Sup), and finally the new CFL-Hybrid limiter (GMCFL-Hyb) detailed in relations (89) to (93). Note that for the sake of comparison, the same scale is used for all figures, even though we did not use equal characteristic sizes $h$ for all grids, as shown in table 2. The order in $L^{1}$ norm of the different schemes is given in table 3 for the six grids.

\begin{tabular}{c|llllll}
\hline Grids & $G_{1}$ & $G_{2}$ & $G_{3}$ & $G_{4}$ & $G_{5}$ & $G_{6}$ \\
\hline FOU & 0.294 & 0.351 & 0.352 & 0.328 & 0.273 & 0.235 \\
LG & 0.714 & 0.690 & 0.758 & 0.723 & 0.807 & 0.729 \\
GM-CFL-Hyb & 0.739 & 0.745 & 0.734 & 0.694 & 0.839 & 0.752 \\
GM-Sup & 0.978 & 0.989 & 0.983 & 0.925 & 1.099 & 0.996 \\
GM-CFL-Sup & 0.957 & 0.973 & 0.985 & 0.883 & 1.112 & 0.967 \\
\hline
\end{tabular}

Table 3: Average schemes order in $L^{1}$ norm on the six grids. Discontinuous case.

As shown by the results, the relative performances of the different schemes can be 

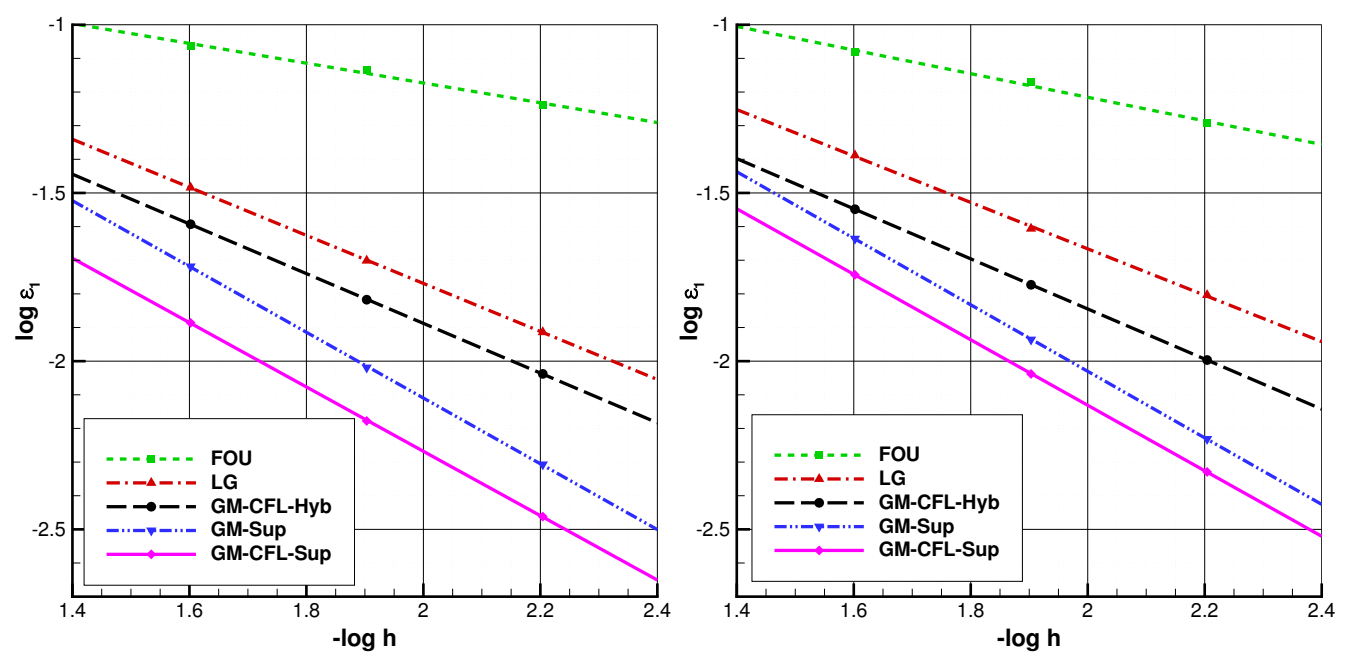

Figure 8: Errors in the $L^{1}$ norm for the discontinuous case on grids $G_{1}$ and $G_{2}$.
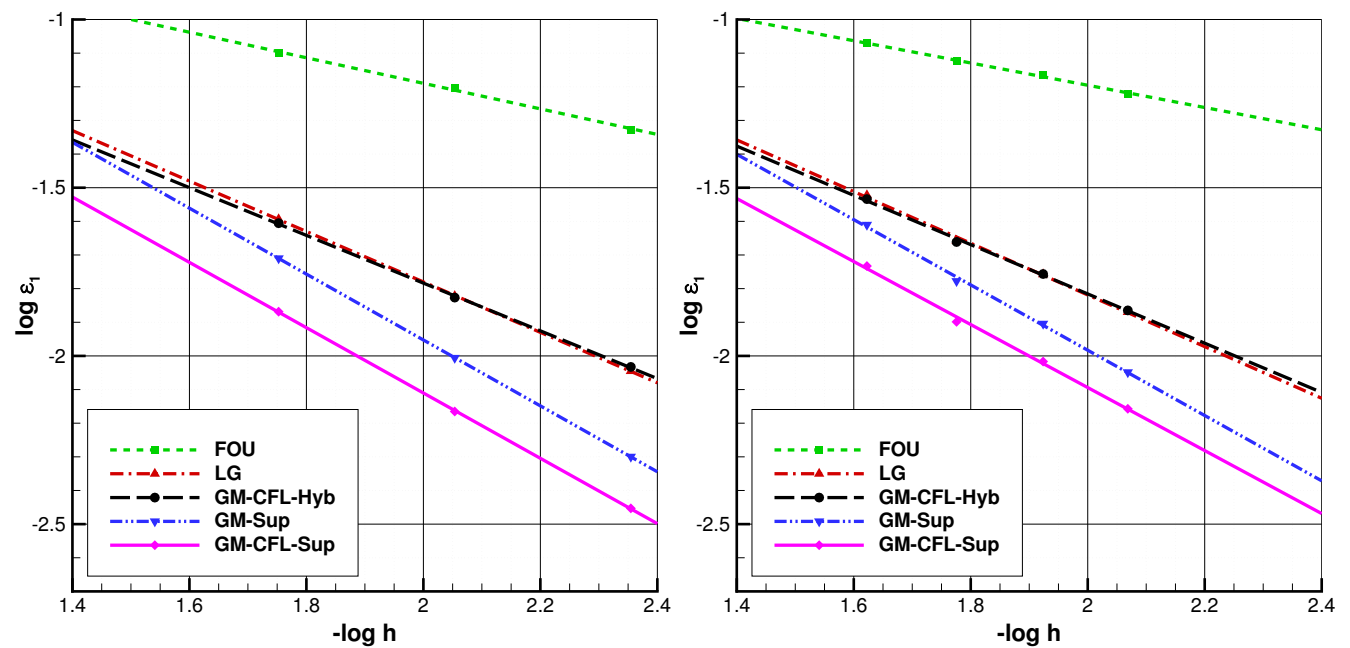

Figure 9: Errors in the $L^{1}$ norm for the discontinuous case on grids $G_{3}$ and $G_{4}$.

classified by increasing accuracy as follows: FOU, LG, GM-CFL-Hyb, GM-Sup, GMCFL-Sup. Since this case is just about convecting a discontinuity, then the results must be interprated only as the shock-capturing efficiency of the different schemes. Consequently, it is natural to get the Superbee-based limiters to be the most accurate, as their compressive behavior is well-known. Moreover GM-CFL-Sup is even more accurate than GM-Sup, which proves the efficiency of introducing the CFL number in the upper bound of the stability region of the limiter.

However, as we already mentioned, Superbee-based limiters are not good candidates 

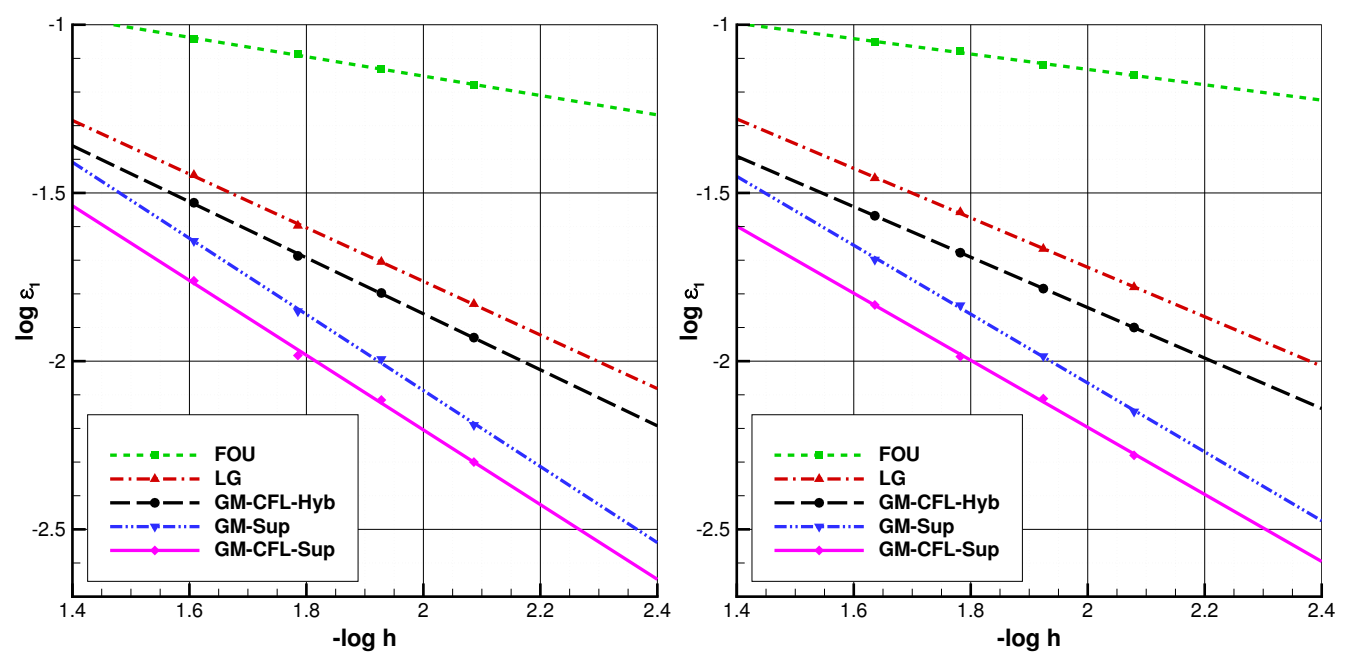

Figure 10: Errors in the $L^{1}$ norm for the discontinuous case on grids $G_{5}$ and $G_{6}$.

on their own for general cases including smooth waves, due to squaring effects. So it is interesting to evaluate the behavior of GM-CFL-Hyb, since we expect it as the best compromise for general cases. And its accuracy is actually better than that of the limited gradient (LG) approach on most grids, except on the triangular grids $G_{3}$ and $G_{4}$ for which we have equal accuracies.

The explanation is as follows: the more edges by element of the grid, in other words the more neighbors for a given element, then the more restrictive the gradient limiting procedures. Consequently, it is natural that LG exhibits extra dissipation on quadrilateral and polygonal meshes, when compared with triangular meshes. However, the scottish grid $G_{2}$, despite being also a triangular mesh, does not offer the same accuracy. The explanation there lies in the mesh curvature, defined as the misalignment of the points $\boldsymbol{B}_{i}, \boldsymbol{M}_{i j}$ and $\boldsymbol{B}_{j}$ (see figure 1). Whereas the grid $G_{3}$ has strictly no curvature and $G_{4}$ has a very small average curvature, the grid $G_{2}$ has a significant curvature. And the accuracy of the gradient technique is lowered for curved meshes, because the interpolations at the faces centroids $\boldsymbol{M}_{i j}$ are not computed with an optimal approximation of the derivative in the direction $\left(\boldsymbol{B}_{i} \boldsymbol{M}_{i j}\right)$.

\subsubsection{Smooth initial solution}

We use the following function as the smooth initial solution:

$$
u_{0}(x, y)=\left\{\begin{array}{ll}
{[1+\cos (4 \pi r)]^{2} / 4} & r \leq 0.25 \\
0 & r>0.25
\end{array} \quad r=\sqrt{(x-0.3)^{2}+(y-0.3)^{2}}\right.
$$

The results for the smooth case on the six grids are shown on figures 11, 12, 13, 14, 15, and 16. We draw linear regressions of the error in $L^{1}$ norm (left hand figures), and in $L^{\infty}$ norm (right hand figures) versus the characteristic size $h$, for four different convective schemes: FOU, LG, and our Generalized Multislope technique equipped with 
the classical CFL-independent Monotonized Central limiter extended to irregular grids (GM-MC), and with the CFL-Hybrid limiter (GM-CFL-Hyb), with the same parameters as in the discontinuous case. The order in $L^{1}$ norm and in $L^{\infty}$ norm of the different schemes is given for the six grids in tables 4 and 5 respectively.
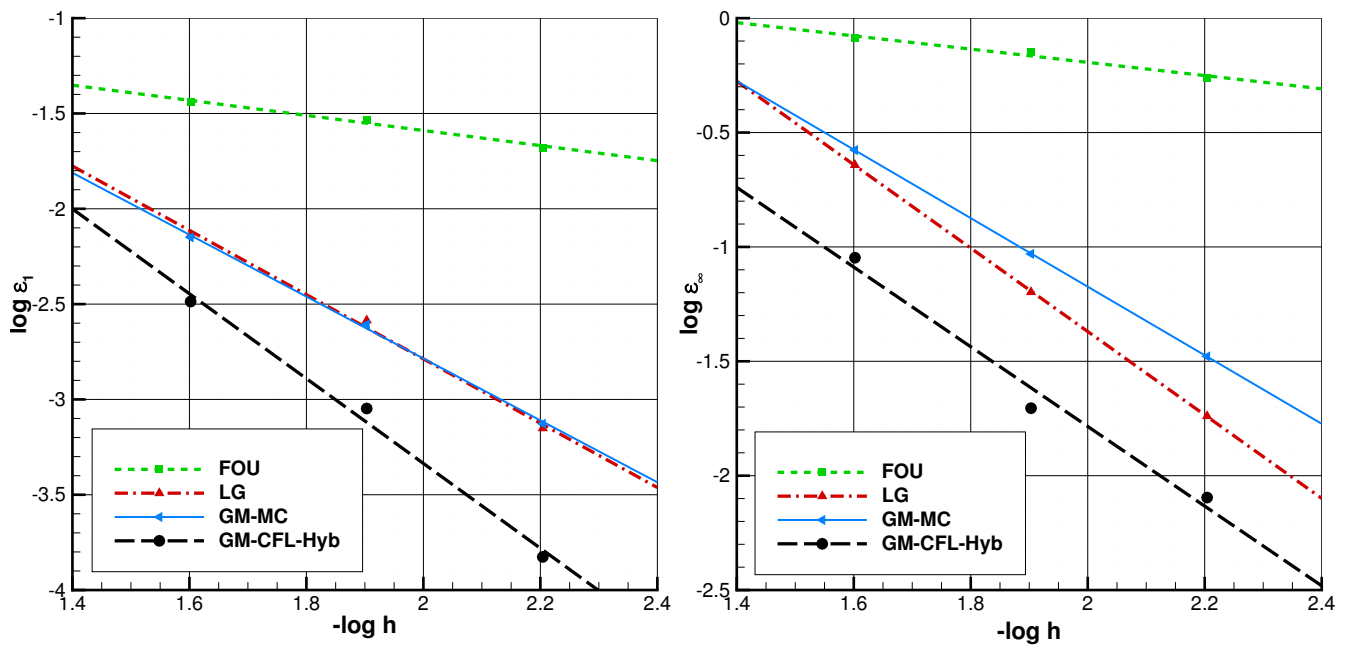

Figure 11: Errors in the $L^{1}$ and $L^{\infty}$ norm for the smooth case on grid $G_{1}$.
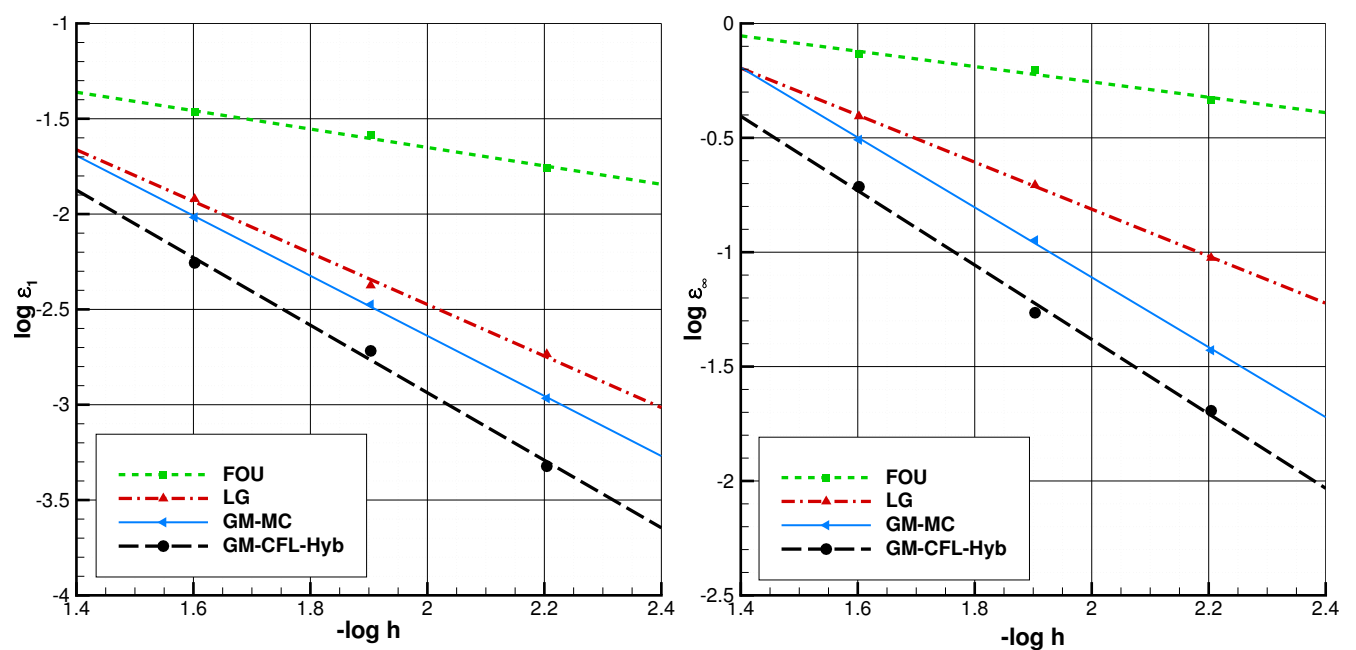

Figure 12: Errors in the $L^{1}$ and $L^{\infty}$ norm for the smooth case on grid $G_{2}$.

The results for the error in $L^{1}$ norm show trends similar to that of the discontinuous case. Indeed, GM-CFL-Hyb is the most accurate for grids $G_{1}, G_{2}, G_{5}$ and $G_{6}$, but is overtaken by LG for grids $G_{3}$ and $G_{4}$. As previously stated, this is explained by both 

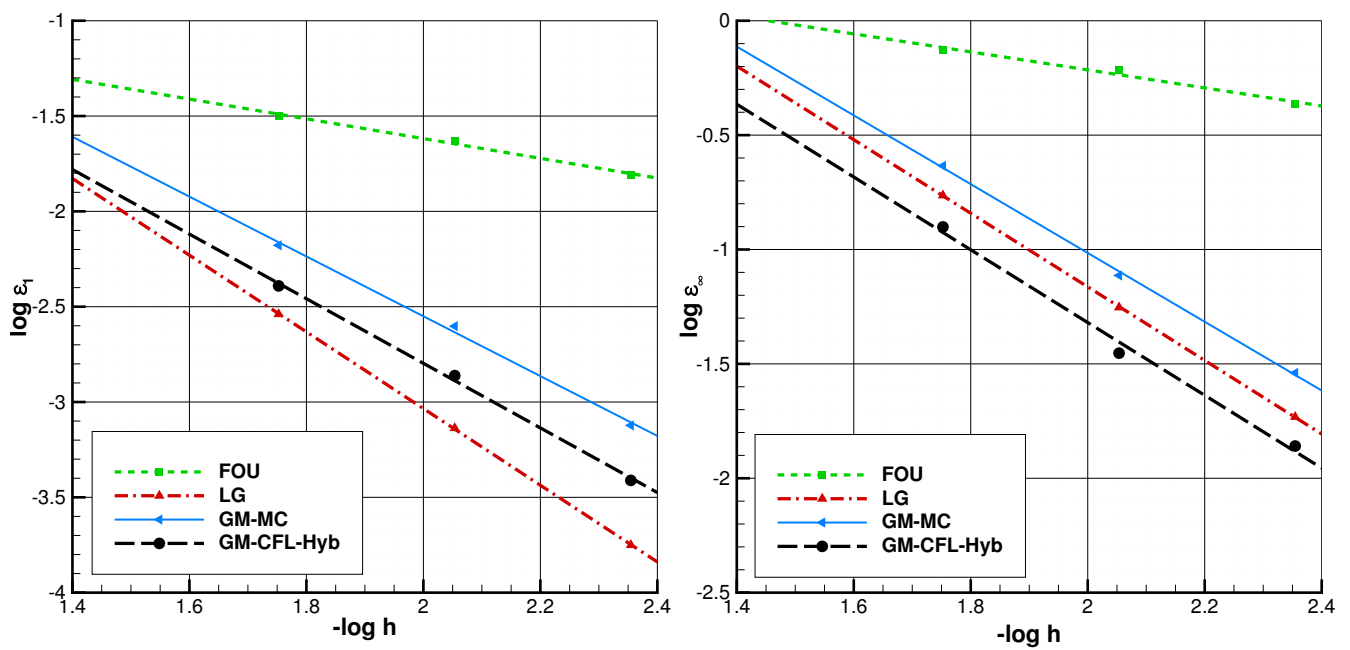

Figure 13: Errors in the $L^{1}$ and $L^{\infty}$ norm for the smooth case on grid $G_{3}$.
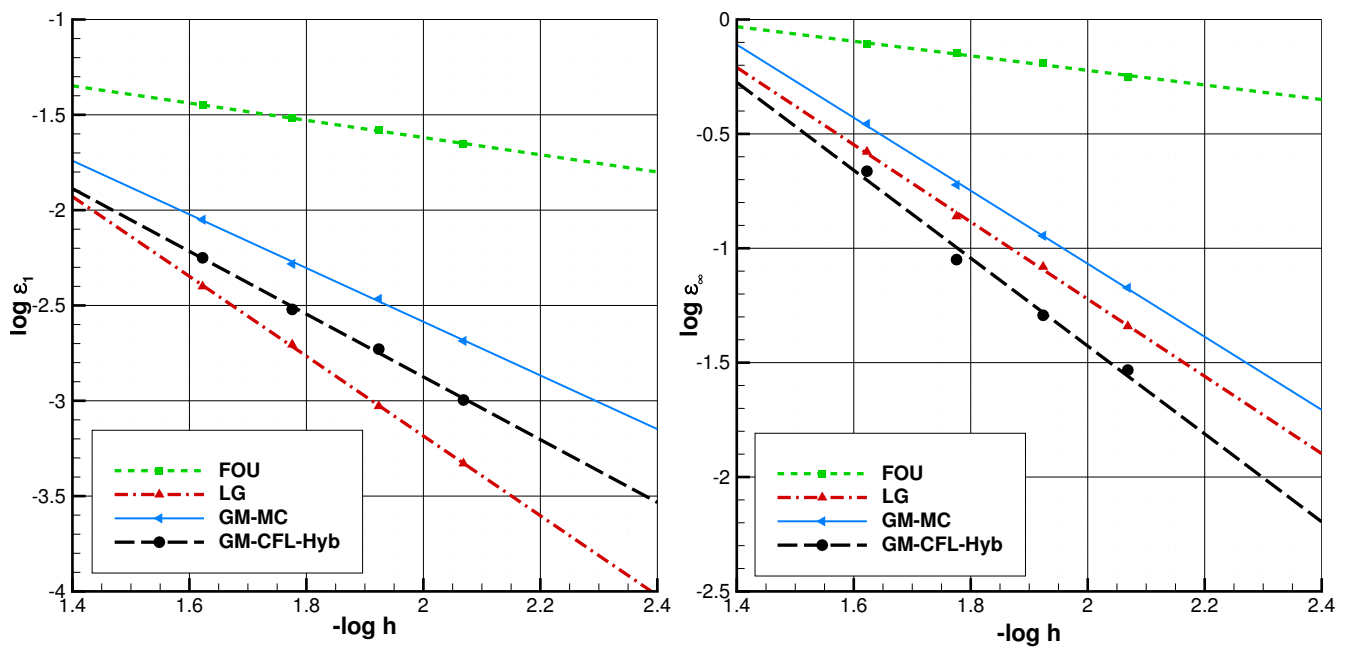

Figure 14: Errors in the $L^{1}$ and $L^{\infty}$ norm for the smooth case on grid $G_{4}$.

the curvature of the grids, and the number of edges of the mesh elements. Besides, except for the grids $G_{3}$ and $G_{4}$, the multislope technique equipped with just a classical CFL-independent limiter such as the Monotonized Central limiter, has already equivalent accuracy as LG.

Furthermore, the error in $L^{\infty}$ norm provides information on the ability of the different schemes to preserve the amplitude of the waves. On that point, the results show the efficiency of GM-CFL-Hyb over the other schemes. In particular, when compared with the monoslope results $(\mathrm{LG})$, this statement is reinforced when the number of edges per 

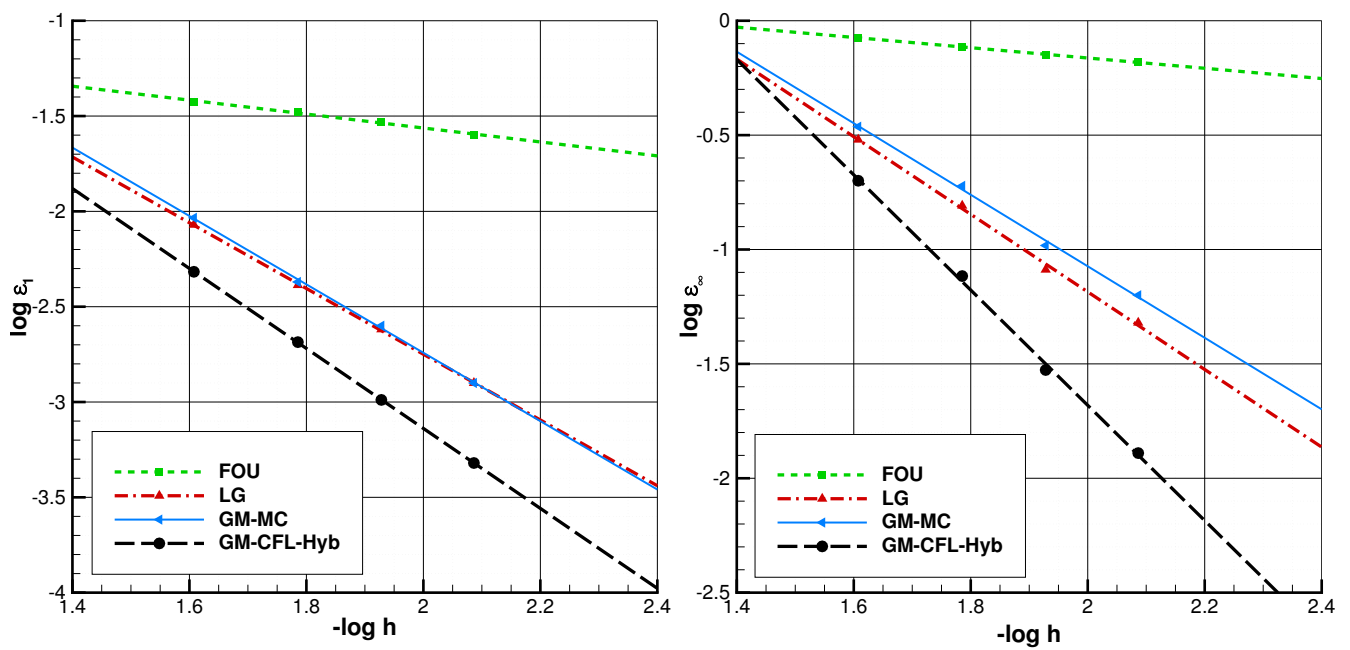

Figure 15: Errors in the $L^{1}$ and $L^{\infty}$ norm for the smooth case on grid $G_{5}$.
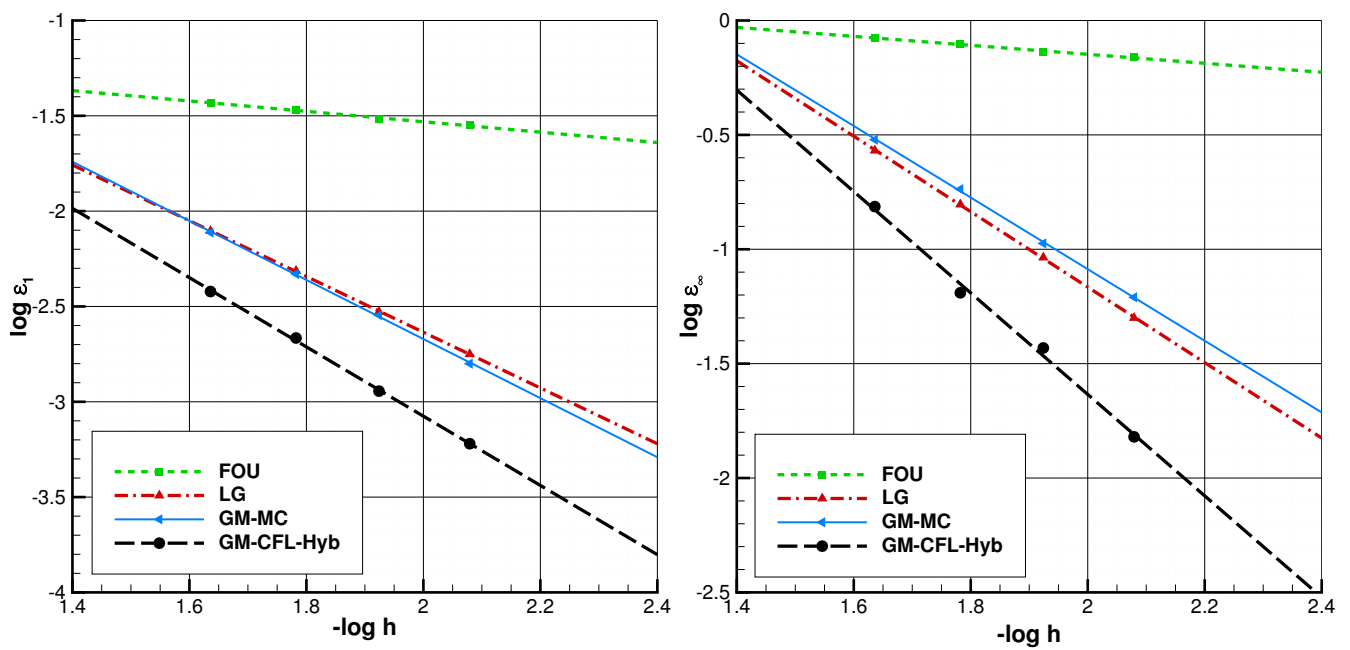

Figure 16: Errors in the $L^{1}$ and $L^{\infty}$ norm for the smooth case on grid $G_{6}$.

element increases $\left(G_{1}, G_{5}, G_{6}\right.$ compared to $G_{3}$ and $\left.G_{4}\right)$, and when strongly curved meshes are involved (grid $G_{2}$ ). Additionally, the comparison between GM-CFL-Hyb and GM-MC proves the significant advantage of the hybridized limiter over a more classical one.

To conclude, the results for both the discontinuous and the smooth cases highlight the major assets of the generalized multislope method:

- it is not limited to a particular kind of mesh. Any unstructered grid can be handled. 


\begin{tabular}{c|llllll}
\hline Grids & $G_{1}$ & $G_{2}$ & $G_{3}$ & $G_{4}$ & $G_{5}$ & $G_{6}$ \\
\hline FOU & 0.396 & 0.483 & 0.518 & 0.462 & 0.331 & 0.292 \\
LG & 1.686 & 1.352 & 2.011 & 2.130 & 1.714 & 1.471 \\
GM-MC & 1.623 & 1.576 & 1.569 & 1.379 & 1.771 & 1.505 \\
GM-CFL-Hyb & 2.225 & 1.771 & 1.694 & 1.622 & 2.096 & 1.813 \\
\hline
\end{tabular}

Table 4: Average schemes order in $L^{1}$ norm on the six grids. Smooth case.

\begin{tabular}{c|llllll}
\hline Grids & $G_{1}$ & $G_{2}$ & $G_{3}$ & $G_{4}$ & $G_{5}$ & $G_{6}$ \\
\hline FOU & 0.201 & 0.200 & 0.309 & 0.319 & 0.198 & 0.185 \\
LG & 1.620 & 0.850 & 1.517 & 1.688 & 1.617 & 1.600 \\
GM-MC & 1.348 & 1.273 & 1.409 & 1.596 & 1.480 & 1.517 \\
GM-CFL-Hyb & 1.851 & 1.497 & 1.613 & 1.921 & 2.297 & 2.184 \\
\hline
\end{tabular}

Table 5: Average schemes order in $L^{\infty}$ norm on the six grids. Smooth case.

- it does not suffer from the local curvature of the mesh as it always take maximum benefit of the available neighborhood to reconstruct the values on the faces.

- it provides better accuracy than a classical monoslope method in most cases, and especially for tricky mesh configurations.

Besides, the results showed the benefits brought by the introduction of the CFL number in the limiters, as well as the hybridization strategy.

\section{3. $3 D$ case}

As exposed in section 3, the algorithm used to determine the points $\boldsymbol{H}_{i j}^{+}$and $\boldsymbol{H}_{i j}^{-}$ is much more complex for 3D configurations since we need to handle many tricky geometrical operations. Therefore, we present in this section a simple 3D case in order to prove the efficiency and operability of the $3 \mathrm{D}$ algorithm. The case herein considered is just an extension in three dimensions of the previous $2 \mathrm{D}$ case. So, we have the following $3 \mathrm{D}$ rotation problem:

$$
\left.\left.\partial_{t} u(x, y, z, t)+\lambda(x, y, z) \cdot \nabla u(x, y, z, t)=0 \quad(x, y, z) \in \Omega=[0,1]^{3}, \quad t \in\right] 0, T\right] .
$$

The divergence-free velocity field reads in

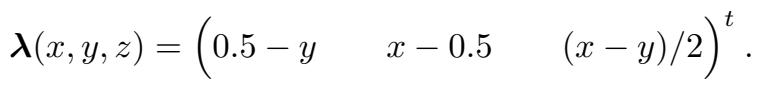

We consider the following smooth initial solution:

$$
\begin{gathered}
u_{0}(x, y, z)=\left\{\begin{array}{ll}
{[1+\cos (4 \pi r)]^{2} / 4} & r \leq 0.15 \\
0 & r>0.15
\end{array},\right. \\
r=\sqrt{(x-0.2)^{2}+(y-0.2)^{2}+(z-0.2)^{2}},
\end{gathered}
$$


which is convected for one revolution, i.e. a simulation time of $T=2 \pi$. The initial 605 explicit RK2 time-stepping scheme. The time step is set to $\Delta t=T / 2000=\pi / 1000 \mathrm{~s}$. We consider the mesh shown on figure 17, made up with unstructured hexahedra. The characteristic size of the mesh is defined as

$$
h \approx\left(\frac{|\Omega|}{\mathcal{N}}\right)^{1 / 3} \approx 0.017 \mathrm{~m},
$$

where $|\Omega|=1$ is the volume of the computational domain, and $\mathcal{N} \approx 215000$ is the number of elements in the mesh. We therefore get a maximum CFL number low enough for explicit time-stepping, namely

$$
\nu_{\max }=M \frac{\Delta t}{h} \approx 0.16
$$

with

$$
M=\max _{(x, y, z) \in \Omega}\|\boldsymbol{\lambda}(x, y, z)\| \approx 0.866
$$

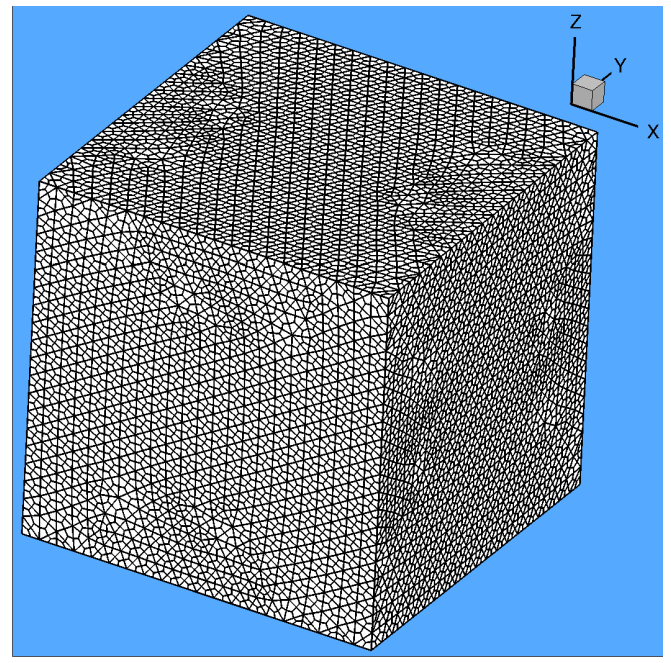

Figure 17: Hexahedral 3D mesh.

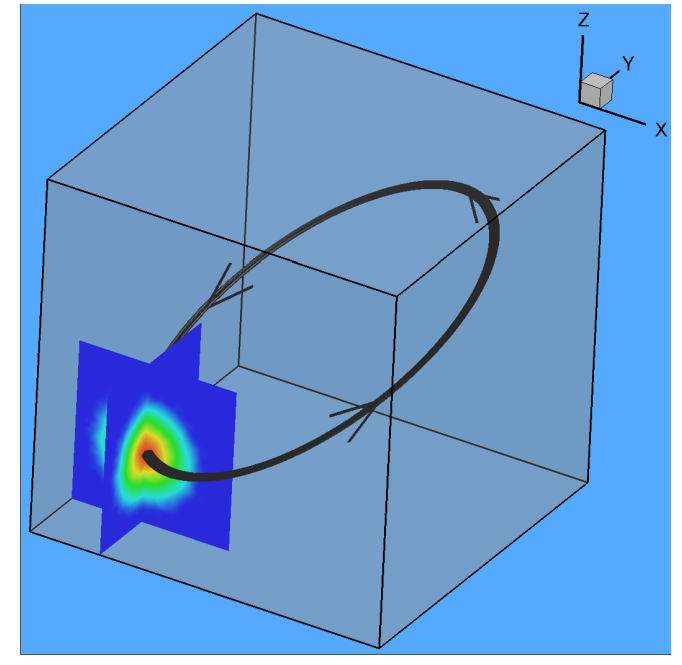

Figure 18: Trajectory of the initial solution.

The errors in $L^{1}$ and $L^{\infty}$ norms, as defined in equations (98) and (99), as well as the max value after one revolution, are given in table 6 for three schemes: the first order upwind (FOU), the monoslope limited gradient (LG), and the generalized multislope with the CFL hybrid limiter (GM-CFL-Hyb). On a qualitative basis, the figures 19, 20, 21 show the respective contours of the final solution in the planes $(X Y),(X Z),(Y Z)$ with either the LG scheme or the GM-CFL-Hyb scheme. These results are in agreement with the trends observed in the previous $2 \mathrm{D}$ cases. The operability and efficiency of the generalized multislope method on a 3D general unstructured mesh is therefore shown, as well as its performance over a classical monoslope technique. 

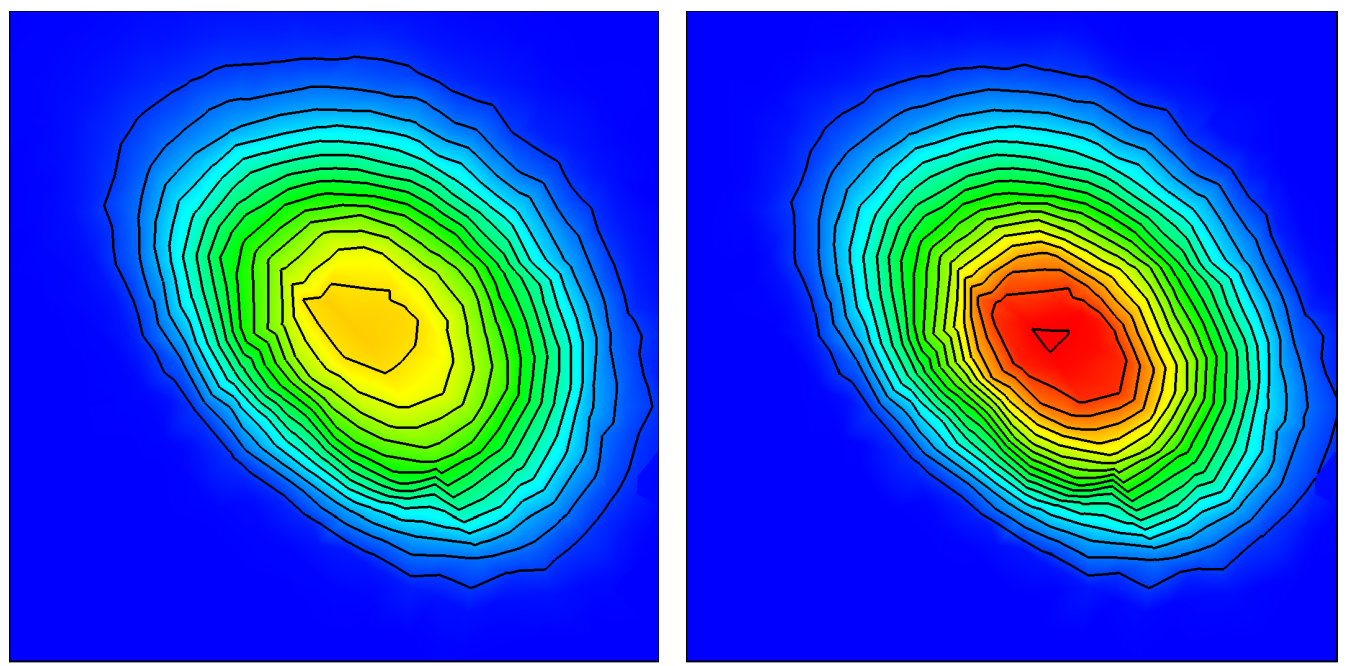

Figure 19: Final solution in the plane ( $X Y)$ with LG (left-hand) and GM-CFL-Hyb (right-hand) schemes.
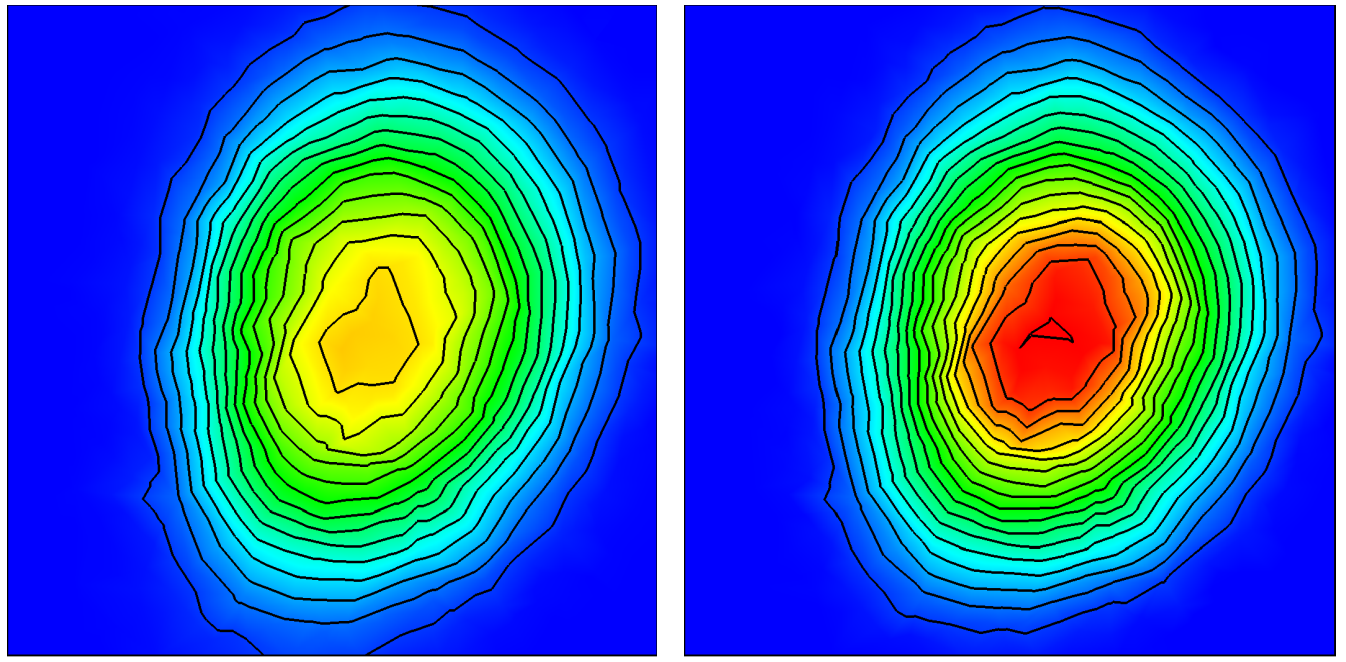

Figure 20: Final solution in the plane $(X Z)$ with LG (left-hand) and GM-CFL-Hyb (right-hand) schemes.

\subsection{Mach 3 wind tunnel with a step}

Let us now apply the generalized multislope procedure to the Euler system of gas dynamics. We consider a classical test case, introduced first in [30], and then used for instance in [31], and more recently with the initial multislope method of [15]. A uniform Mach 3 flow enters a 2D wind tunnel from its left-hand side. The wind tunnel is 3 meters long and 1 meter wide, and a step of 0.2 meter height is located 0.6 meter from the tunnel inlet (see figure 23). The tunnel is initially filled with an ideal gas under the following conditions: $P=1, \rho=1.4, \gamma=1.4, v_{x}=3$. Thus, the initial sound speed is $c_{0}=(\gamma P / \rho)^{1 / 2}=1$. The same gas with the same properties is then continually injected 

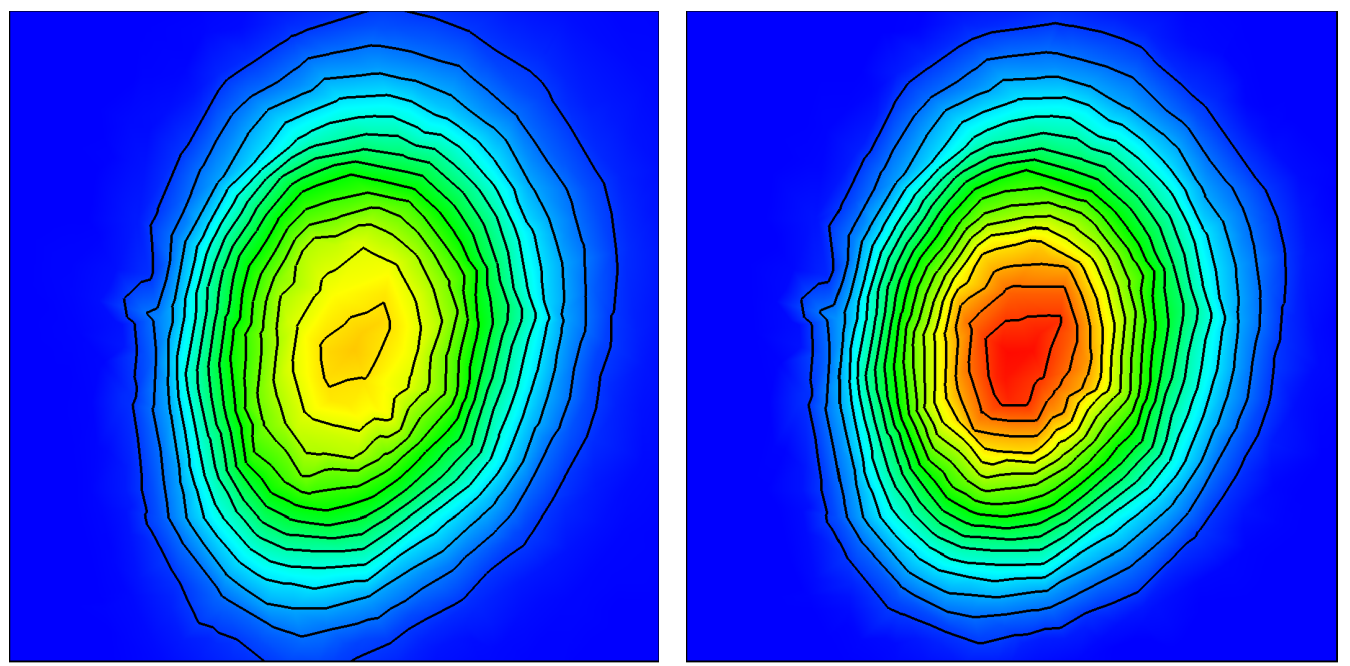

Figure 21: Final solution in the plane $(Y Z)$ with LG (left-hand) and GM-CFL-Hyb (right-hand) schemes.

\begin{tabular}{|c|c|c|c|}
\hline & FOU & LG & GM-CFL-Hyb \\
\hline $\boldsymbol{\epsilon}_{\mathbf{1}}$ & $5.93 \cdot 10^{-3}$ & $2.61 \cdot 10^{-3}$ & $2.24 \cdot 10^{-3}$ \\
\hline $\boldsymbol{\epsilon}_{\boldsymbol{\infty}}$ & 0.943 & 0.528 & 0.509 \\
\hline max value & 0.0664 & 0.527 & 0.652 \\
\hline
\end{tabular}

Table 6: Errors in the $L^{1}$ and $L^{\infty}$ norm for the 3D smooth case.

from the left inlet.

The computation is performed on a hybrid unstructured mesh, a sample of which is depicted on figure 22. The ability of the generalized multislope reconstruction to deal with the Euler system on completely general meshes is thereby assessed. This mesh is made up of 16,836 cells composed of triangular and quadrangular elements. This number of cells is very close to that used in [31] $(16,128)$ with a structured mesh, and in [15] $(16,714)$ with only triangular elements. As in [15], the numerical flux is computed with the HLL approximate Riemann solver of [32], so that no particular treatment is required to deal with the singular point at the step corner.

The multislope reconstruction is applied to the primitive variables $(P, \boldsymbol{v}, \rho)$ to preserve the positivity. Moreover, because the Euler system is not a scalar equation, we have to make clear the definition of the pseudo-CFL term $\nu_{i j}^{+}$we have included in the CFL hybrid limiter. In the stability demonstration for the scalar case, this term was defined as (see (45) in section 4.2)

$$
\nu_{i j}^{+}=\Delta t \frac{\left|S_{i j}\right|}{\left|K_{i}\right|} \frac{\partial \phi_{i j}}{\partial U_{1}}\left(\widetilde{U_{i j}}, U_{i}\right),
$$

and it is not clear how to extend this definition for a non scalar system. Our choice is to 
take the wave of maximum amplitude which can actually cross the face, which means

$$
\nu_{i j}^{+}=\Delta t \frac{\left|S_{i j}\right|}{\left|K_{i}\right|}\left(\left|\boldsymbol{v}_{i} \cdot \boldsymbol{n}_{i j}\right|+c_{i}\right),
$$

with $\boldsymbol{v}_{i}$ the velocity vector at the cell center, $\boldsymbol{n}_{i j}$ the outwards unit normal vector of the face $S_{i j}$, and $c_{i}$ the sound speed at the cell center.

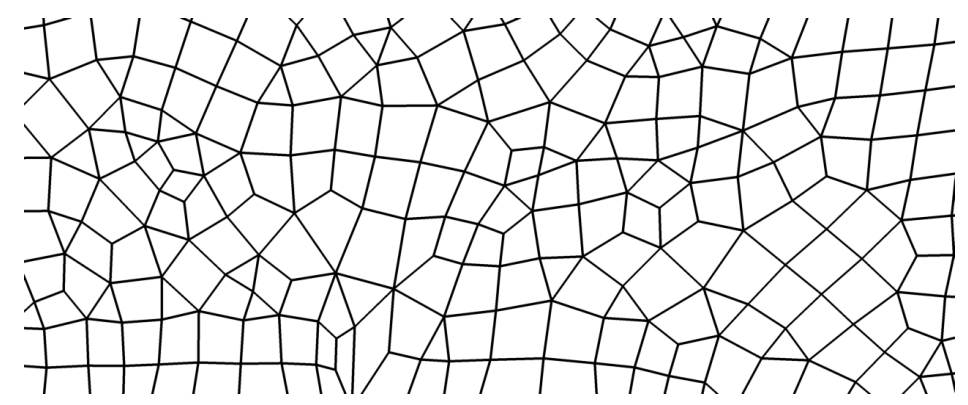

Figure 22: Sample of the hybrid unstructured mesh used for the Mach 3 wind tunnel test case.

The results of this case using the first order upwind scheme (FOU), the monoslope limited gradient (LG), and the generalized multislope method with the CFL hybrid limiter (GM-CFL-Hyb), are respectively depicted on figures 23,24 , and 25 . The figure 26 shows the results using the GM-CFL-Hyb on a much finer mesh, made up with approximately 100,000 elements. These results can therefore be used as a reference, when comparing the schemes behaviors on the coarser grid. All the figures include thirty density isolines, ranging from 0.25 to 6 . As in [31] and [15], these are shown at the time $t=4$.

These results were obtained with an explicit RK2 time-stepping scheme, and the time step has been set to $\Delta t \approx 5 \cdot 10^{-4}$ for the coarse mesh, and $\Delta t \approx 2.5 \cdot 10^{-4}$ for the fine mesh. Moreover, if

$$
h_{i}=4\left|K_{i}\right| / \sum_{j \in \mathcal{V}(i)}\left|S_{i j}\right|
$$

stands for the size of a given element $K_{i}$, then $h_{0}=\min _{K_{i}} h_{i}$ denotes the minimum element size over the whole domain. We actually have $h_{0} \approx 5 \cdot 10^{-3}$ for the coarse mesh, and $h_{0} \approx 2.5 \cdot 10^{-3}$ for the fine mesh, which therefore entails for both meshes an initial maximum CFL number of

$$
\nu_{\max }=M \frac{\Delta t}{h_{0}} \approx 0.4, \quad \text { with } \quad M=\max _{\substack{K_{i} \\ t=0}}\left(\left\|\boldsymbol{v}_{i}\right\|+c_{i}\right)=4 .
$$

The comparison of figures 23, 24 and 25 first shows that better accuracy is achieved with the second-order schemes. The use of the GM-CFL-Hyb scheme also gives a better resolution of the contour lines when compared with the monoslope method. We also note that these results are of slightly better quality than those obtained by [15] on a mesh made up with only triangular elements. Moreover, we use the face gravity centre for the reconstructions ensuring true second-order accuracy, while in [15], the computations may fail when using this point. 


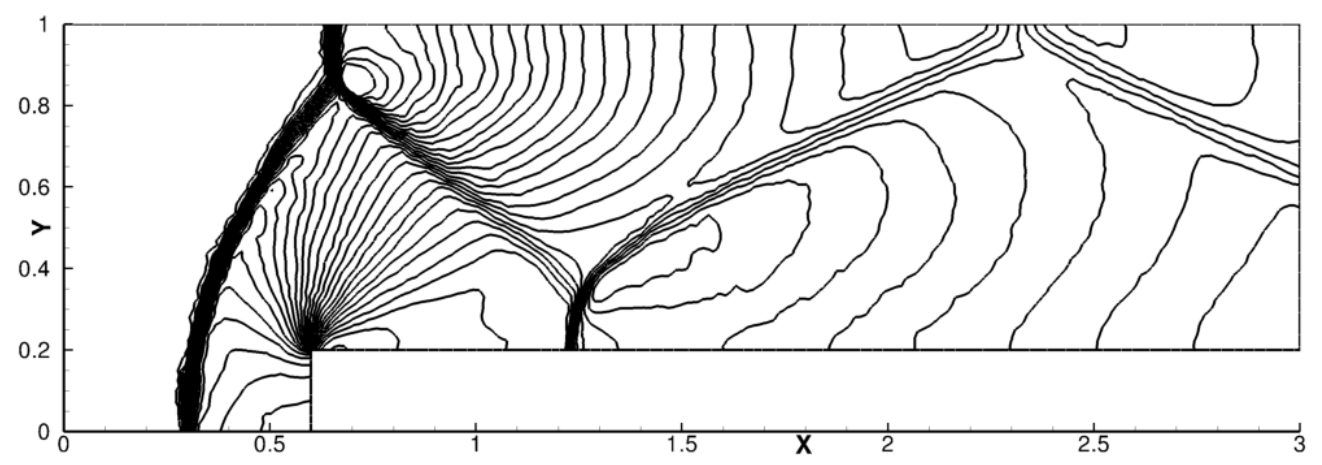

Figure 23: FOU scheme, coarse mesh.

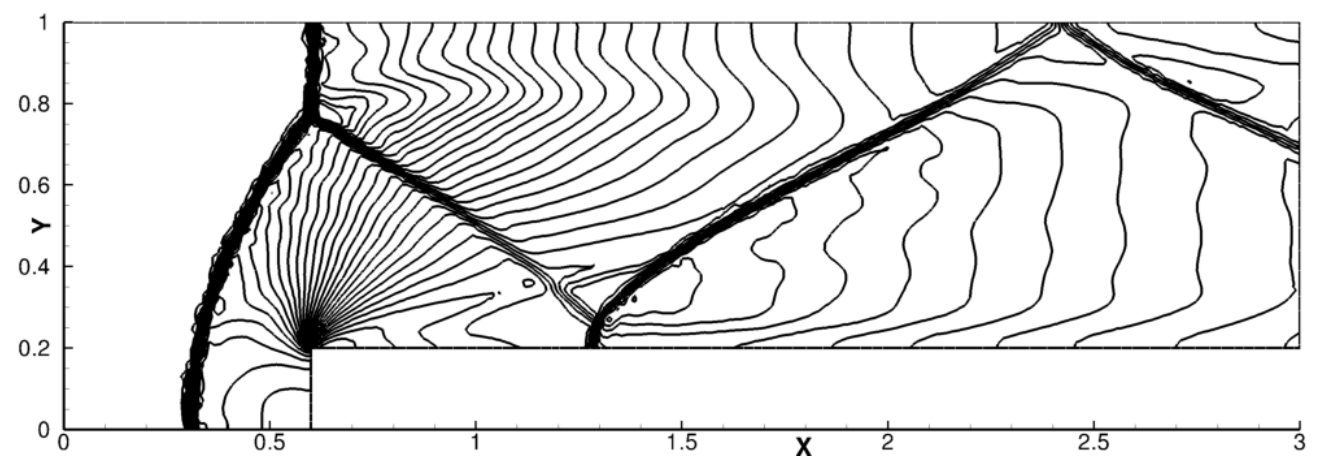

Figure 24: LG scheme, coarse mesh.

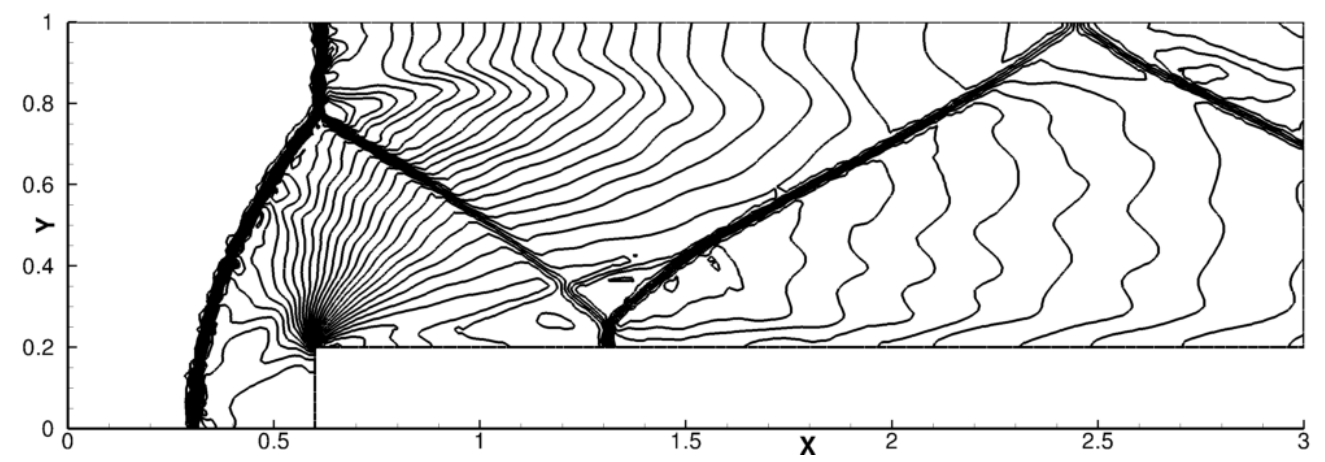

Figure 25: GM-CFL-Hyb scheme, coarse mesh.

\subsection{Liquid water jet case}

We now consider a much more complex test case, involving a two-phase flow configuration. A liquid water jet is injected at the velocity $20 \mathrm{~m} / \mathrm{s}$, with a pressure of 1 bar and a temperature of $300 \mathrm{~K}$, into quescient air at the same pressure and temperature. This 675 case is modeled by the multi-species compressible Navier-Stokes equations (see Appendix 


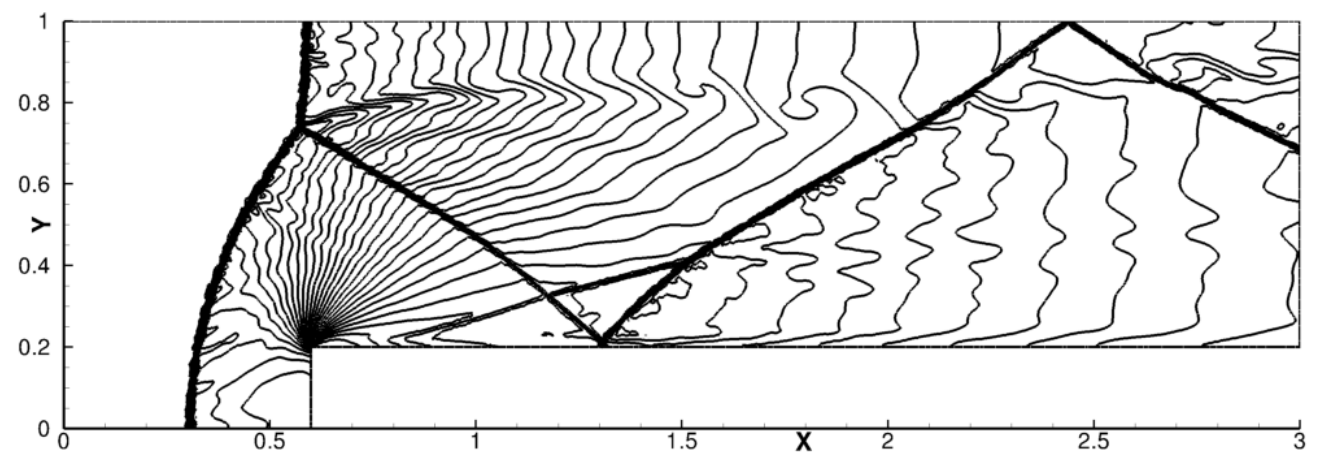

Figure 26: GM-CFL-Hyb scheme, fine mesh.

$\mathrm{C}$ for more details on the equations). Liquid water and air are therefore the two species of one single fluid, which is locally described by unique homogeneous density, pressure, velocity and temperature. This means that there is no specific treatment to describe the topology of the liquid-gas interface. There is no turbulence modelling either. The objective here is only to assess the ability of the reconstruction procedures to preserve stability and accuracy in spite of the high density ratio involved at the interface.

We use a hybrid unstructured mesh, made up with approximately 138,000 arbitrary polygonal elements (see figure 27). Numerical fluxes are computed with the HLLC approximate Riemann solver. A second-order explicit time-stepping scheme is used, and the time step is set to $\Delta t=10^{-5} \mathrm{~s}$, which implies the following maximum CFL number:

$$
\nu_{\max } \approx \Delta t \max _{K_{i}}\left(\frac{\left|S_{i j}\right|}{\left|K_{i}\right|}\right)\left(\max _{K_{i}}\left\|\boldsymbol{v}_{i}\right\|+\max _{K_{i}} c_{i}\right) \approx 0.3,
$$

with a minimum cell diameter of $0.05 \mathrm{~m}$, and a maximum speed sound of $1415 \mathrm{~m} / \mathrm{s}$ in pure water. Density contours of the liquid-gas mixture obtained with the first order upwind scheme (FOU), and the generalized multislope method with the CFL hybrid

${ }_{690}$ limiter (GM-CFL-Hyb) are shown on figure 28 at the time $t=1.95 \mathrm{~s}$. Figure 29 shows the contours of liquid water mass fraction obtained with these two schemes at the same time.

The comparison between the FOU and GM-CFL-Hyb schemes shows clearly the increase of accuracy brought about by the multislope procedure. In particular, the liquid-

${ }_{695}$ gas interface can hardly be seen in the first-order computation, while the multislope scheme provides a sharp definition of the interface. Besides, the classical monoslope method we have used for the previous tests has also been tested on this case. However the computation fails, even with a time step lowered to $\Delta t=2 \cdot 10^{-6} \mathrm{~s}$, which means a maximum CFL number around 0.06. Strong pressure oscillations arise in the course

700 of the computation, until it fails due to a negative pressure. It is not clear why this monoslope method is not robust on this case, and we have not tested all the monoslope procedures available. Nevertheless, we experienced no particular difficulty with regard to stability using the generalized multislope procedure on this test case, while being able to efficiently capture many structures of the flow. 


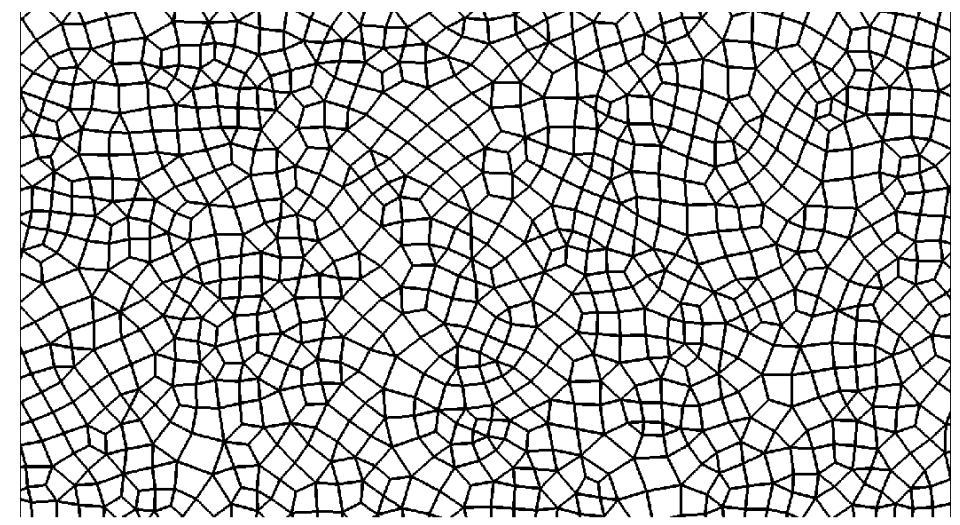

Figure 27: Sample of the hybrid unstructured mesh used for the liquid water jet case. vature or polyhedral elements. Furthermore, the multislope formalism enables a genuine upwind treatment of the reconstruction procedure. Added to upwind numerical fluxes, this therefore seems a suitable way to ensure robustness for complex applications involving high density ratios, large discontinuities, or shocks.

This method has been implemented within the industrial CEDRE code, developed at ONERA [19]. More specifically, it has been integrated in a compressible Navier-Stokes solver, and in a solver dedicated to the eulerian description of dispersed two-phase flows [33]. The test cases performed in this paper on complex flows involving shocks and very high density ratios has proved the robustness and accuracy of this method when 725 compared with classical monoslope techniques.

This generalized multislope method has also been successfully used for applications in space propulsion involving two-phase flows and turbulent combustion and the results have been presented elsewhere [34]. In these computations, the classical monoslope approaches may fail while the presented technique enabled us to solve robustness issues inherent to 730 two-phase flows, mainly due to the existence of strong discontinuities or vacuum. This makes this new multislope technique a promising tool for robust and accurate simulations of combustion and two-phase flow problems, in the cell-centered finite volume formalism. 

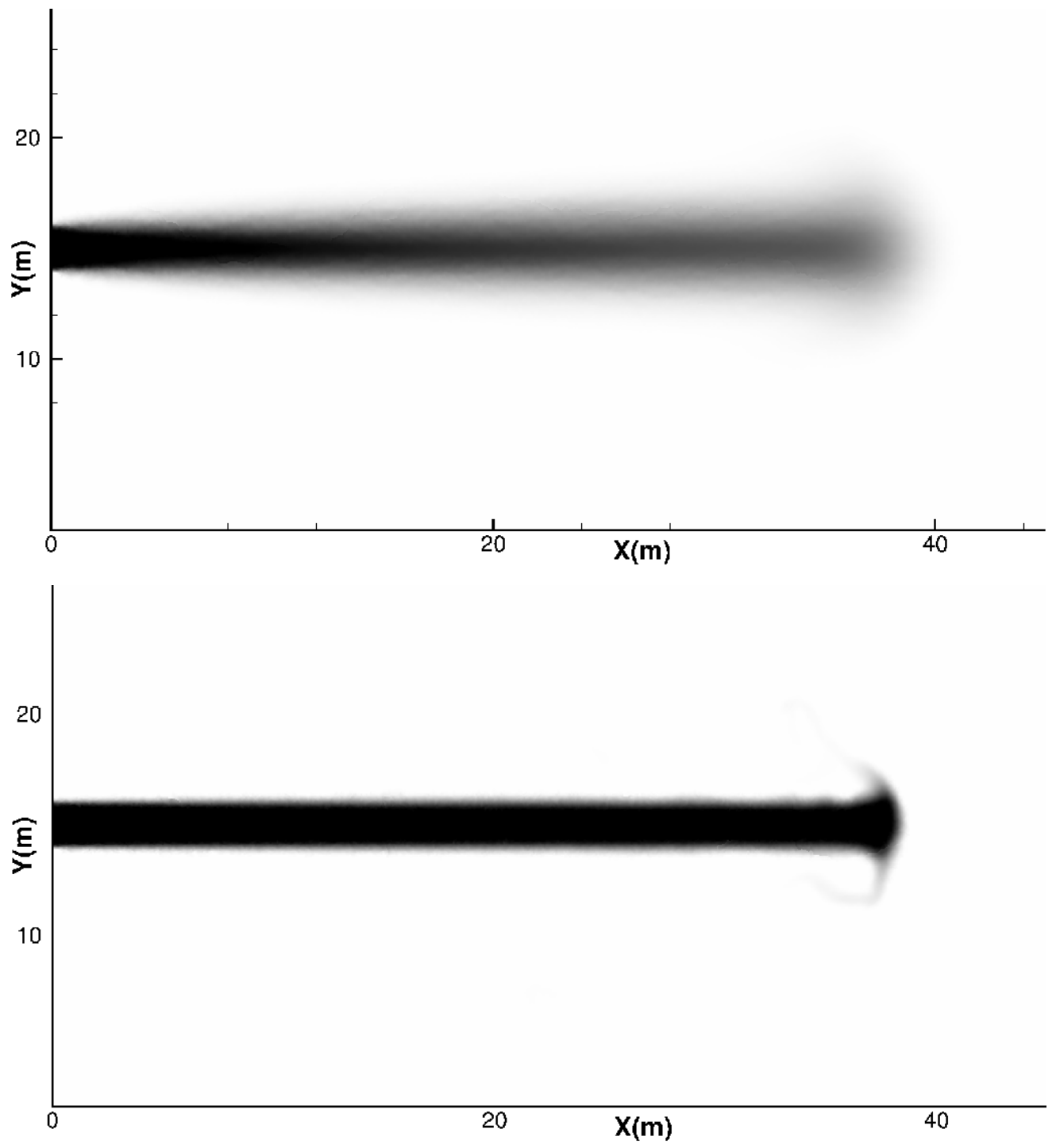

Figure 28: Liquid-gas mixture density contours with the FOU scheme (top) and the GM-CFL-Hyb scheme (bottom). Min value: $\rho_{\min }=1.17$. Max value: $\rho_{\max }=999.8$.

\section{Acknowledgements}

This work was supported by a PhD grant from ONERA - The French Aerospace Lab. 735 The authors also acknowledge the very valuable comments of one of the referees that greatly helped to improve the presentation and clarity of the paper. 

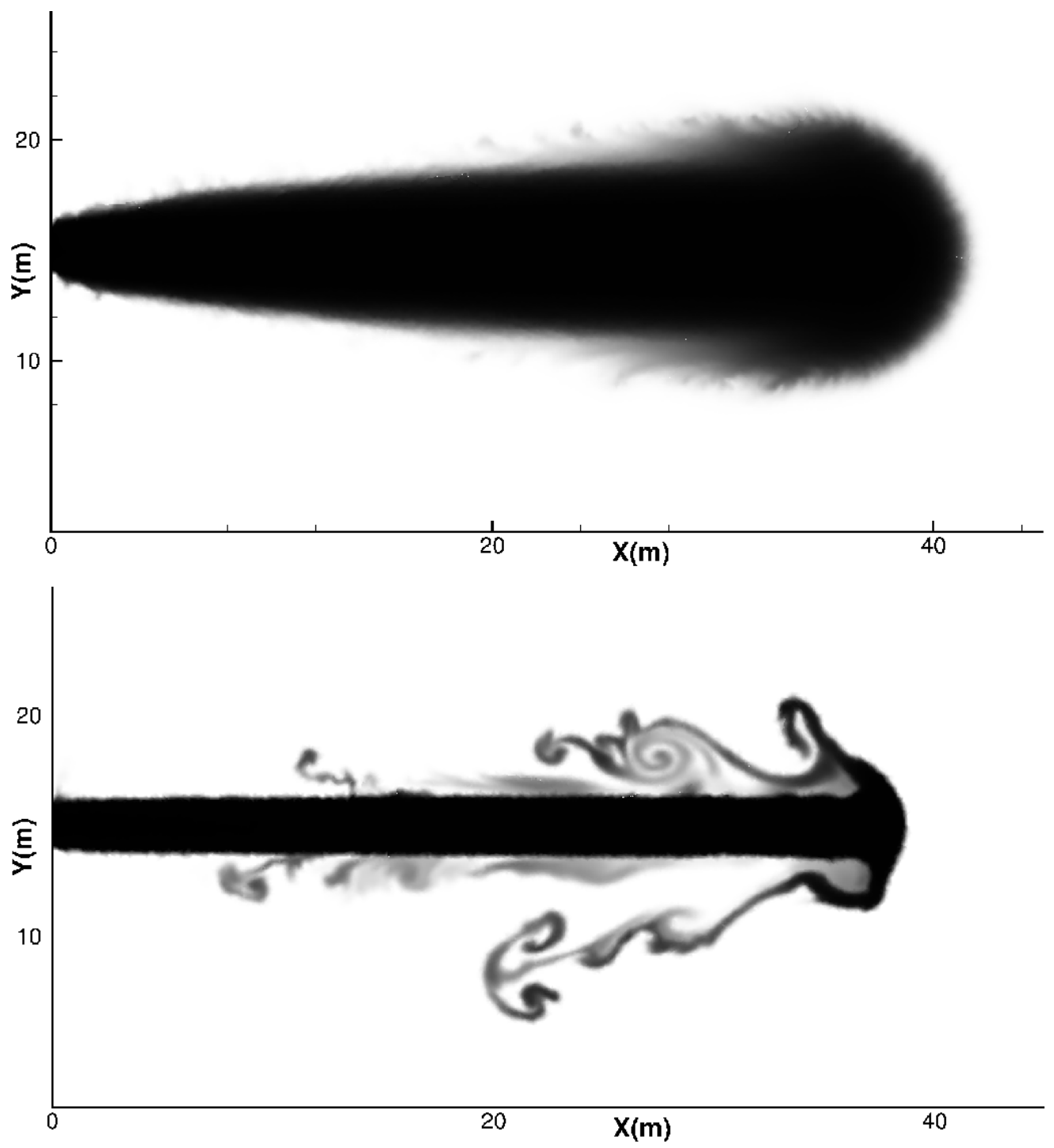

Figure 29: Liquid water mass fraction contours with the FOU scheme (top) and the GM-CFL-Hyb scheme (bottom).

Appendix A. Proof of the second-order accuracy of the values $U_{H_{i j}^{+}}$and $U_{H_{i j}^{-}}$

Let us assume a linear variation of $U$ across the domain $\Omega$. Therefore, the following expression holds for any $\boldsymbol{x}$ and $\boldsymbol{x}_{0} \in \Omega$ :

$$
U(\boldsymbol{x})=U\left(\boldsymbol{x}_{0}\right)+\nabla U \cdot\left(\boldsymbol{x}-\boldsymbol{x}_{0}\right),
$$


740 where $\nabla U$ denotes the uniform gradient of $U$. According to equations (17) to (22), we can write for both the $2 \mathrm{D}$ and the $3 \mathrm{D}$ formalisms, with $d=2$ or $d=3$ respectively,

$$
U_{H_{i j}^{+}}=\sum_{l=1}^{d} \beta_{i j_{l}}^{+} U_{i j_{l}}^{+}=\sum_{l=1}^{d} \beta_{i j_{l}}^{+} U_{i}+\sum_{l=1}^{d} \beta_{i j_{l}}^{+}\left(U_{i j_{l}}^{+}-U_{i}\right) .
$$

Using formula (A.1), and because $\sum_{l=1}^{d} \beta_{i j_{l}}^{+}=1$, we can rewrite

$$
U_{H_{i j}^{+}}=U_{i}+\nabla U \cdot \sum_{l=1}^{d} \beta_{i j_{l}}^{+} \boldsymbol{B}_{i} \boldsymbol{B}_{i j_{l}}^{+}
$$

and by decomposition of the vector $\boldsymbol{B}_{i} \boldsymbol{B}_{i j_{l}}^{+}$, it follows

$$
U_{H_{i j}^{+}}=U_{i}+\nabla U \cdot \boldsymbol{B}_{i} \boldsymbol{H}_{i j}^{+} \sum_{l=1}^{d} \beta_{i j_{l}}^{+}+\nabla U \cdot \sum_{l=1}^{d} \beta_{i j_{l}}^{+} \boldsymbol{H}_{i j}^{+} \boldsymbol{B}_{i j_{l}}^{+} .
$$

By property of the barycentre, we have

$$
\sum_{l=1}^{d} \beta_{i j_{l}}^{+} \boldsymbol{H}_{i j}^{+} \boldsymbol{B}_{i j_{l}}^{+}=\mathbf{0}
$$

which leads to

$$
U_{H_{i j}^{+}}=U_{i}+\nabla U \cdot \boldsymbol{B}_{i} \boldsymbol{H}_{i j}^{+}=U\left(\boldsymbol{H}_{i j}^{+}\right)
$$

and completes the proof. A similar result is straightforwardly obtained for $U_{H_{i j}^{-}}$:

$$
U_{H_{i j}^{-}}=U\left(\boldsymbol{H}_{i j}^{-}\right)
$$

\section{Appendix B. Proof of the property of the hybrid limiter}

According to (90), we have

$$
\varphi^{\prime}(r)=\varphi_{1}^{\prime}(r)+h^{\prime}(r)\left[\varphi_{2}(r)-\varphi_{1}(r)\right]+h(r)\left[\varphi_{2}^{\prime}(r)-\varphi_{1}^{\prime}(r)\right] .
$$

Besides, $h(0)=1, \varphi_{1}(0)=\varphi_{2}(0)=0$. So the derivative in $0^{+}$reads in

$$
\varphi^{\prime}(0)=\varphi_{1}^{\prime}(0)+\varphi_{2}^{\prime}(0)-\varphi_{1}^{\prime}(0)=\varphi_{2}^{\prime}(0) .
$$

750 Now considering the derivative in $r=1$, and because $h(1)=0, \varphi_{1}(1)=\varphi_{2}(1)=1$, we have

$$
\varphi^{\prime}(1)=\varphi_{1}^{\prime}(1)+h^{\prime}(1)[1-1]+0=\varphi_{1}^{\prime}(1)
$$

which completes the proof. 


\section{Appendix C. Navier-Stokes compressible equations for the liquid jet case}

We consider a "two-phase" homogeneous fluid described by the Navier-Stokes compressible equations (C.1), where $\boldsymbol{Q}(\boldsymbol{U})$ is the vector of the conservative variables defined in (C.2). These are $\rho Y_{g}$ and $\rho Y_{l}$, the mass densities of the gazeous and liquid species, $\rho \boldsymbol{v}$ the momentum vector, and $e_{\text {tot }}$ the total energy which is the sum of internal and kinetic energies.

$$
\begin{gathered}
\frac{\partial \boldsymbol{Q}(\boldsymbol{U})}{\partial t}+\nabla \cdot\left[\boldsymbol{F}_{c}(\boldsymbol{U})-\boldsymbol{F}(\boldsymbol{U}, \nabla \boldsymbol{U})\right]=0 \\
\boldsymbol{Q}(\boldsymbol{U})=\left(\begin{array}{llll}
\rho Y_{g} & \rho Y_{l} & \rho \boldsymbol{v} & \rho e_{t o t}
\end{array}\right)^{t}, \quad \rho=\rho Y_{g}+\rho Y_{l}
\end{gathered}
$$

From the conserved quantities $\boldsymbol{Q}$, it is possible to describe the physical state of the fluid with the vector of "natural" variables (C.3), where $P$ and $T$ are the homogeneous fluid pressure and temperature, $\boldsymbol{v}$ is the fluid velocity vector, $Y_{g}$ and $Y_{l}$ are the mass fractions of the gas and liquid species.

$$
\boldsymbol{U}(\boldsymbol{Q})=\left(\begin{array}{lllll}
P & T & \boldsymbol{v} & Y_{g} & \left.Y_{l}\right)^{t},
\end{array} Y_{g}+Y_{l}=1\right.
$$

Thermodynamical mixture laws provide the system closure. The Navier-Stokes system (C.1) comprises the convective fluxes (C.4), $\boldsymbol{I}_{3}$ being the unit tensor and the diffusive fluxes (C.5).

$$
\begin{aligned}
\boldsymbol{F}_{c}(\boldsymbol{U}) & =\boldsymbol{Q} \otimes \boldsymbol{v}+P\left(\begin{array}{llllll}
0 & \ldots & 0 & \boldsymbol{I}_{3} & \boldsymbol{v}
\end{array}\right)^{t} \\
\boldsymbol{F}(\boldsymbol{U}, \nabla \boldsymbol{U}) & =\left(\begin{array}{llllll}
F_{\rho Y_{1}} & \ldots & F_{\rho Y_{n_{g}}} & F_{\rho Y_{l}} & F_{v} & F_{e}
\end{array}\right)^{t}
\end{aligned}
$$

The mass diffusive fluxes $F_{\rho Y_{i}}$ are modeled by Fick's law. The total energy flux gathers the heat fluxes, partial enthalpy fluxes, and the power of the viscous forces. The momentum flux $F_{v}$ is linked to the viscous stress tensor by $F_{v}=-\tau_{\text {lam }}-\tau_{\text {tur }}$, where $\tau_{\text {lam }}$ is the laminar stress tensor and $\tau_{\text {tur }}$ is the turbulent stress tensor, which vanishes here since we do not consider any turbulence modeling.

\section{References}

[1] B. van Leer, Towards the ultimate conservative difference scheme. V. A second-order sequel to Godunov's method, Journal of Computational Physics 32 (1) (1979) 101136. doi:10.1016/0021-9991(79)90145-1.

URL http://linkinghub.elsevier.com/retrieve/pii/0021999179901451

[2] E. Godlewski, P.-A. Raviart, Numerical Approximation of Hyperbolic Systems of Conservation Laws, Springer, New York, NY, 1996.

[3] T. Barth, M. Ohlberger, Finite volume methods: Foundation and analysis, in: Encyclopedia of Computational Mechanics, Volume 1, Fundamentals, John Wiley and Sons Ltd, 2004, p. 439-474. 
[4] S. K. Godunov, A difference method for numerical calculation of discontinuous solutions of the equations of hydrodynamics, Matematicheskii Sbornik 89 (3) (1959) $271-306$.

URL http://www . mathnet.ru/eng/sm4873

[5] S. Osher, Convergence of generalized MUSCL schemes, SIAM Journal on Numerical Analysis 22 (5) (1985) 947-961. doi:10.1137/0722057. URL http://epubs.siam.org/doi/abs/10.1137/0722057

[6] P. K. Sweby, High resolution schemes using flux limiters for hyperbolic conservation laws, SIAM Journal on Numerical Analysis 21 (5) (1984) 995-1011. doi:10.1137/ 0721062.

URL http://epubs.siam.org/doi/abs/10.1137/0721062

[7] A. Harten, High resolution schemes for hyperbolic conservation laws, Journal of Computational Physics 49 (3) (1983) 357-393. doi:10.1016/0021-9991(83) 90136-5.

URL http://linkinghub.elsevier.com/retrieve/pii/0021999183901365

[8] P. Colella, Multidimensional upwind methods for hyperbolic conservation laws, Journal of Computational Physics 87 (1) (1990) 171-200. doi:10.1016/0021-9991(90) 90233-Q.

URL http://linkinghub.elsevier.com/retrieve/pii/002199919090233Q

[9] J. B. Goodman, R. J. LeVeque, On the accuracy of stable schemes for 2D scalar conservation laws, Mathematics of Computation 45 (171) (1985) 15. doi:10.2307/ 2008046 .

URL http://www . jstor .org/stable/2008046?origin=crossref

[10] S. Spekreijse, Multigrid solution of monotone second-order discretizations of hyperbolic conservation laws, Mathematics of Computation 49 (179) (1987) 135. doi : $10.2307 / 2008254$. URL http://www . jstor .org/stable/2008254?origin=crossref

[11] A. Jameson, Analysis and design of numerical schemes for gas dynamics 1 Artificial diffusion, upwind biasing, limiters and their effect on accuracy and multigrid convergence, International Journal of Computational Fluid Dynamics 4 (1995) 171-218.

[12] M. Hubbard, Multidimensional slope limiters for MUSCL-type finite volume schemes on unstructured grids, Journal of Computational Physics 155 (1) (1999) 54-74. doi:10.1006/jcph.1999.6329.

URL http://linkinghub.elsevier.com/retrieve/pii/S0021999199963295

[13] B. Stoufflet, J. Periaux, F. Fezoui, A. Dervieux, Numerical simulation of 3-D hypersonic euler flows around space vehicles using adapted finite elements, American Institute of Aeronautics and Astronautics, 1987. doi:10.2514/6.1987-560. URL http://arc.aiaa.org/doi/abs/10.2514/6.1987-560

[14] P.-H. Cournède, C. Debiez, A. Dervieux, A positive MUSCL scheme for triangulations, Tech. Rep. RR-3465, INRIA (Jul. 1998). URL http://hal .inria.fr/inria-00073225 
[15] T. Buffard, S. Clain, Monoslope and multislope MUSCL methods for unstructured meshes, Journal of Computational Physics 229 (10) (2010) 3745-3776. doi:10. $1016 / j \cdot j c p .2010 .01 .026$.

URL http://linkinghub.elsevier.com/retrieve/pii/S0021999110000495

[16] C. Calgaro, E. Chane-Kane, E. Creusé, T. Goudon, L $\infty$-stability of vertex-based MUSCL finite volume schemes on unstructured grids: Simulation of incompressible flows with high density ratios, Journal of Computational Physics 229 (17) (2010) 6027-6046. doi:10.1016/j.jcp.2010.04.034.

URL http://linkinghub.elsevier.com/retrieve/pii/S0021999110002214

[17] V. Clauzon, Analyse de schémas d'ordre élevé pour les écoulements compressibles. Application à la simulation numérique d'une torche à plasma., Ph.D. thesis, Université Blaise Pascal, Clermont-Ferrand (2008).

[18] A. Murrone, H. Guillard, Behavior of upwind scheme in the low Mach number limit: III. Preconditioned dissipation for a five equation two phase model, Computers \& Fluids 37 (10) (2008) 1209-1224. doi:10.1016/j.compfluid.2006.12.010. URL http://linkinghub.elsevier.com/retrieve/pii/S0045793008000054

[19] A. Refloch, CEDRE software, AerospaceLab Journal 2 (2011) 131-140.

[20] G. Grospellier, B. Lelandais, The Arcane development framework, ACM Press, 2009, pp. 1-11. doi:10.1145/1595655.1595659.

URL http://portal.acm.org/citation. cfm?doid=1595655.1595659

[21] B. Courbet, C. Benoit, V. Couaillier, F. Haider, M. C. Le Pape, S. Péron, Space discretization methods, AerospaceLab Journal 2 (2011) 66-79.

[22] N. Leterrier, Discrétisation spatiale en maillage non-structuré de type général, Ph.D. thesis, Université Pierre et Marie Curie (Paris VI) (2003).

[23] S. Clain, V. Clauzon, L $\infty$ stability of the MUSCL methods, Numerische Mathematik 116 (1) (2010) 31-64. doi:10.1007/s00211-010-0299-2. URL http://link.springer.com/10.1007/s00211-010-0299-2

[24] M. Arora, P. L. Roe, A well-behaved TVD limiter for high-resolution calculations of unsteady flow, Journal of Computational Physics 132 (1) (1997) 3-11. doi: $10.1006 / j \mathrm{cph} .1996 .5514$. URL http://linkinghub.elsevier.com/retrieve/pii/S002199919695514X

[25] M. Berger, M. J. Aftosmis, S. M. Murman, Analysis of slope limiters on irregular grids, AIAA paper 490 (2005) 1-22.

URL http://www.nas.nasa.gov/assets/pdf/techreports/2005/nas-05-007. pdf

${ }_{850}^{85}$ [26] F. Kemm, A comparative study of TVD-limiters - well-known limiters and an introduction of new ones, International Journal for Numerical Methods in Fluids 67 (4) (2011) 404-440. doi:10.1002/fld.2357.

URL http://doi.wiley.com/10.1002/fld.2357 
[27] N. Waterson, H. Deconinck, Design principles for bounded higher-order convection schemes - a unified approach, Journal of Computational Physics 224 (1) (2007) 182207. doi:10.1016/j.jcp.2007.01.021.

URL http://linkinghub.elsevier.com/retrieve/pii/S002199910700040X

[28] F. Haider, Discretization on general unstructured grids and applications on LES, Theses, Université Pierre et Marie Curie - Paris VI (May 2009). URL https://tel.archives-ouvertes.fr/tel-00813512

[29] P. O'Rourke, M. Sahota, A variable explicit/implicit numerical method for calculating advection on unstructured meshes, Journal of Computational Physics 143 (2) (1998) 312-345. doi:10.1006/jcph.1998.5903.

URL http://linkinghub.elsevier.com/retrieve/pii/S0021999198959034

[30] A. F. Emery, An evaluation of several differencing methods for inviscid fluid flow problems, Journal of Computational Physics 2 (3) (1968) 306-331. doi:10.1016/ 0021-9991 (68) 90060-0.

URL http://linkinghub.elsevier.com/retrieve/pii/0021999168900600

[31] P. Woodward, P. Colella, The numerical simulation of two-dimensional fluid flow with strong shocks, Journal of Computational Physics 54 (1) (1984) 115-173. doi: 10.1016/0021-9991(84)90142-6.

URL http://linkinghub.elsevier.com/retrieve/pii/0021999184901426

[32] A. Harten, P. D. Lax, B. van Leer, On upstream differencing and Godunov-type schemes for hyperbolic conservation laws, SIAM Review 25 (1) (1983) 35-61. doi: $10.1137 / 1025002$.

URL http://epubs.siam.org/doi/abs/10.1137/1025002

[33] A. Murrone, P. Villedieu, Numerical modeling of dispersed two-phase flows, AerospaceLab Journal 2 (2011) 34-46.

[34] C. L. Le Touze, A. Murrone, E. Montreuil, H. Guillard, Eulerian numerical methods on unstructured meshes for the large eddy simulation of sprays within liquid rocket engines, in: Proceedings of ECCOMAS 2012, 2012. 